\title{
Graphical Contents List
}

Gelatin-pectin composite films from polyion complex hydrogels

Stefano Farris ${ }^{1,}$, Karen M. Schaich ${ }^{2}$, LinShu Liu $^{3}$, Peter H. Cooke ${ }^{3}$, Luciano Piergiovanni ${ }^{1}$, Kit L. Yam $^{2}$

${ }^{1}$ Department of Food Science and Microbiology - Packaging laboratory

University of Milan Via Celoria, 2 - 20133 Milan, Italy

${ }^{2}$ Department of Food Science, Rutgers University, 65 Dudley Road,

New Brunswick, New Jersey 08901, USA

${ }^{3}$ Eastern Regional Research Center, United States Department of

Agriculture, 600 East Mermaid Lane, Wyndmoor, Pennsylvania

19038, USA

*Corresponding Author. Tel: +390250316654; fax: +39 0250316672

E-mail address: stefano.farris@ unimi.it (S. Farris)

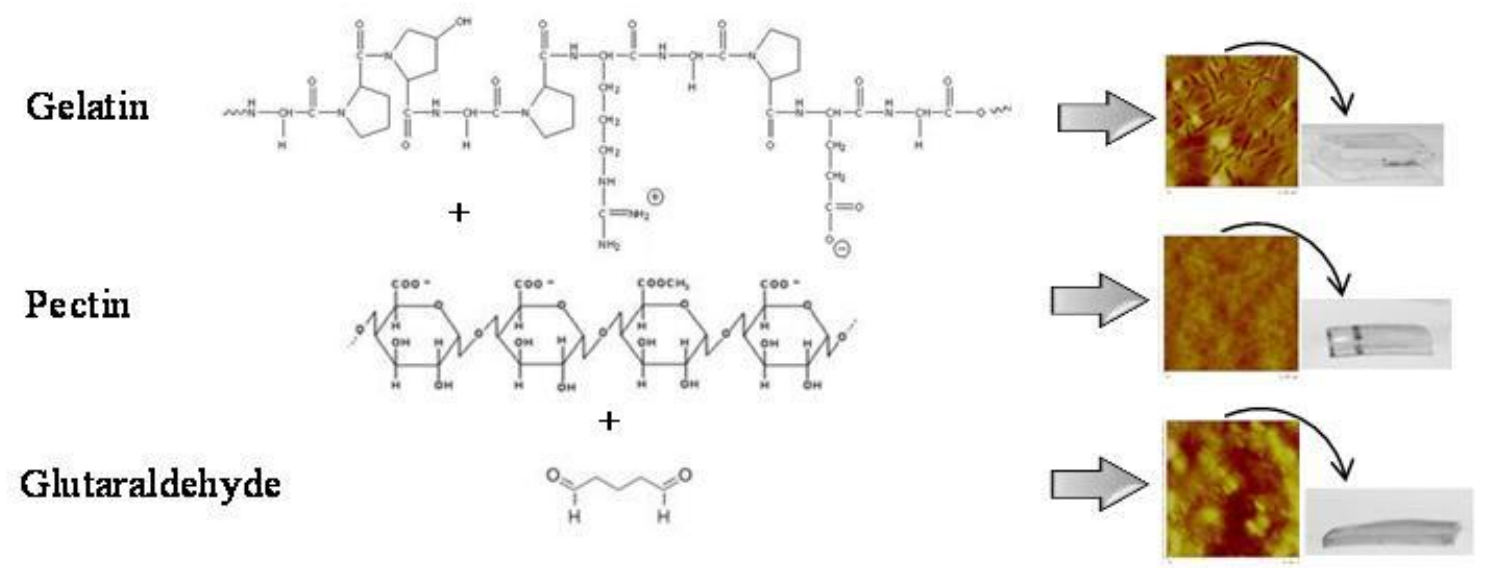




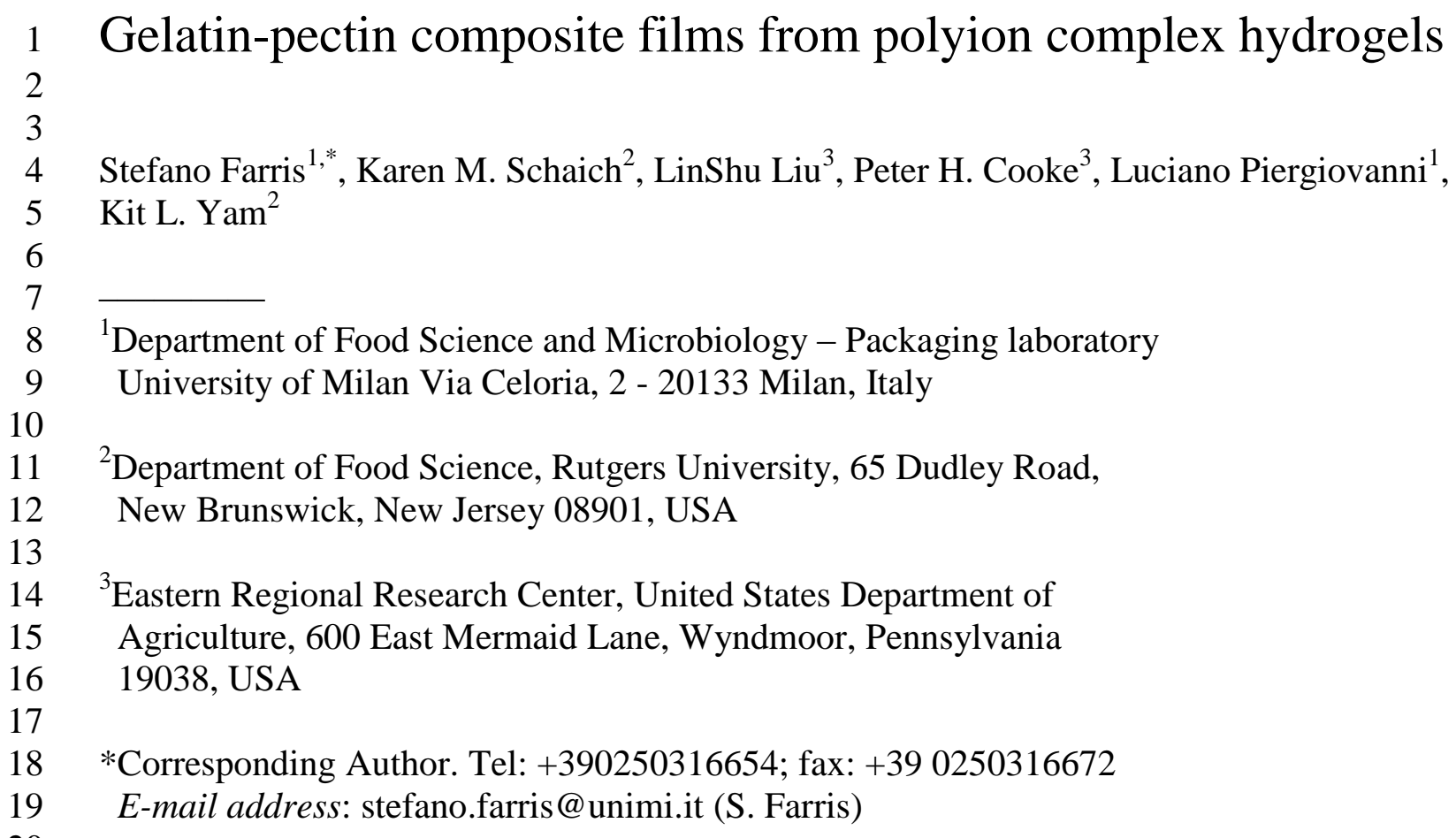

\section{ABSTRACT}

Preparation and properties of composite films from gelatin and low-methoxyl pectin from simultaneous reversible and permanent polyion complex hydrogels are presented. Ionic interactions between positively charged gelatin and negatively charged pectin produce reversible physical hydrogels with homogeneous molecular arrangement that improve both mechanical and water resistance but do not alter thermal stability relative to single polymer gels. Subsequent addition of 0.3 weight percent (wt.-\%) glutaraldehyde crosslinks gelatin heterogeneously, due to the presence of domains with non-uniform crosslinking, as revealed by the structural analysis. Resulting interspersed permanent chemical hydrogel showed a decreased swelling attitude by nearly 10 fold relative to films from gelatin alone and further improved mechanical performance (tensile strength and elongation at break). Results demonstrate that simultaneously exploiting the specific reactivity provided by the functional groups of both biopolymers can be used to create unique new structures with improved properties and offer potential for tailoring these to a wide range of targeted applications.

Keywords: AFM; crosslinking; electrostatic interactions; hydrogel films; SEM 


\section{Introduction}

The past few years have witnessed rapidly expanding interest in renewable agricultural

feedstocks and marine food processing wastes as sources of biomolecules with potential to replace synthetic polymers (Tharanathan, 2003) in fabricating biomaterials with bioactivity, biocompatibility, biodegradability, and novel properties for unique applications (Lin \& Metters, 2006). As biomolecules have become more available, ever-increasing demand for high-performance "natural" matrices for biomedical and pharmaceutical applications such as tissue engineering (Khademhosseini \& Langer, 2007) and organ regeneration (Skotak, Leonov, Larsen, Noriega, \& Subramanian, 2008; Jaklenec, Wan, Murray, \& Mathiowitz, 2008; Lai, Lu, Chen, Tabata, \& Hsiue, 2006), controlled drug delivery systems (Ghaffari, Navaee, Oskoui, Bayati, \& Rafiee-Tehrani, 2007; Wei, Sun, Wu, Yin, \& Wu, 2006; Kurisawa \& Yui, 1998), bioadhesives for wound dressing (Ong, Wu, Moochhala, Tan, \& Lu, 2008), films, contact lenses, and capsules for oral ingestion (Hoffman et al., 2002) has stimulated design of 'smart' matrices able to 'sense' external changes ( $\mathrm{pH}$, temperature, humidity) and trigger release of active (drugs) and/or bioactive (protein and genes) compounds (Jeong, Kim, $\&$ Bae, 2002). Although applications are less developed, these same properties offer promise for use of biopolymers in packaging materials as stand-alone films (Mohareb \& Mittal, 2007; Weber, Haugaard, Festersen, \& Bertelsen, 2002) and as thin layers that either carry active compounds to be released into a targeted environment or provide a coating to improve the properties of a base film (Gong, Katsuyama, Kurokawa, \& Osada, 2003).

In spite of well-established benefits, biomaterials still suffer some drawbacks that hinder full exploitation. Most widely recognized limitations include mechanical weakness (and thus inability to withstand loads) (Farris, Introzzi, \& Piergiovanni, 2009a), water sensitivity (which leads to unwanted matrix failures) (Yao, Liu, Chang, Hsu, \& Chen, 2004), and instability under physiological conditions with unpredictable behaviour in long-term applications (Lin et al., 2006). Although different strategies may be pursued to overcome these problems, the 
inherent versatility and multifunctionality of such biomolecules offer a valuable opportunity to improve their physicochemical and biochemical properties, thus affording new possibilities for specific applications (Mourya \& Inamdar, 2008; Ravi Kumar, 2000). Accordingly, one way to improve the overall performance of bio-based materials is represented by the association of biomolecules of both different origin and chemical characteristics through the development of new methods/techniques, which makes it possible to fully exploit the reactivity of functional groups along the skeleton of biomolecules (Hoare \& Kohane, 2008). Protein-polysaccharide pairs, in particular, have great potential to fabricate many structural complexes and coacervates with improved physicochemical properties, exploitable for films/coatings-forming purposes (Turgeon, Schmitt, \& Sanchez, 2007; de Kruif \& Tuinier, 2001; Wooster \& Augustin, 2007; Giancone, Torrieri, Masi, \& Michon, 2009). Among the various combinations, low-methoxyl pectin and gelatin have been described as well-suited hydrocolloids for producing simple hydrogels (Liu, Liu, Fishman, \& Hicks, 2007; Nikolova, Panchev, \& Sainova, 2005; Gilsenan, Richardson, \& Morris, 2003). Recently, low-methoxyl pectin and gelatin have also been indicated as valuable candidate to generate new elaborate architectures originating from a multi-step approach (Farris, Schaich, Liu, Piergiovanni, \& Yam, 2009b).

This paper provides experimental evidence for formation of new materials from integrated hydrogel networks using gelatin and low-methoxyl pectin as reactive biopolymers. It is documented the qualitative molecular structures as well as some main quantitative physical properties of films generated from sequential combination of hydrogels solutions of low-methoxyl pectin and gelatin. To this purpose, a 2-step approach has been adopted, according to the experimental procedure we proposed recently in the attempt of obtaining new high-performance materials defined as 'permanent polyion-complex hydrogels' films (Farris et al., 2009b). The rationale behind the experimental work here presented is thoroughly documented elsewhere (Farris et al., 2009b). Briefly, in a first step, solutions of gelatin and 
low-methoxyl pectin are combined to generate a continuous physical co-gel in which the minor component (low-methoxyl pectin) is dispersed through the interstices of the original main network (gelatin). In this mixed matrix, gelatin and pectin interact with each other through electrostatic forces, yielding a reversible physical polyion complex that has better overall performance than either individual polymer. In a second step, the inclusion of glutaraldehyde in this polyion complex chemically crosslinks gelatin, giving rise to a permanent hydrogel complex that improves strength and water-resistance still further. This step is effective because the chemical groups of gelatin involved in the ionic interactions with pectin differ from those forming covalent bonds with glutaraldehyde.

Results demonstrate how progressively increasing the complexity of molecular interactions in biopolymer hydrogels (simple gelatin hydrogels $\rightarrow$ physical gelatin-pectin hydrogels $\rightarrow$ permanent polyion complexes) can be used creatively to produce flexible films that have tremendously enhanced performances (e.g., strength and moisture resistance). This provides compelling support for using biopolymers from renewable resources to synthesize strong films with desirable properties.

\section{Experimental}

\subsection{Source of materials}

Type A, 250 Bloom, pharmaceutical and food grade pigskin gelatin powder (isoelectric point, IEP 9.0): Weishardt International, Grauliet Cedex, France. Low-methoxyl (DE = 7, $\mathrm{pK}_{\mathrm{a}} \sim 3.5$; native $\mathrm{pH}$ ) pectin: CP Kelco, San Diego, CA. Glycerol (plasticizer): Giomavaro, Brugherio, Italy. Glutaric dialdehyde (25 wt.-\% in water): Acros, Morris Plane, NJ. All reagents were used as received, without further purification. All solutions were prepared with Milli-Q water (18.3 M $\Omega)$. 


\subsection{Preparation of the films}

116 Three types of films with $21 \mathrm{wt} \%$ total solids but different composition were generated

117 mixing gelatin, pectin, and glycerol in different amount as settled in our previous work (Farris

118 et al., 2009b). Gelatin hydrogels (gel) (native $\mathrm{pH} \sim 4.5$ ) were prepared by mixing gelatin

119 powder (14 wt.-\%) with water containing 7.0 wt.- $\%$ glycerol, heating to $60 \pm 0.5{ }^{\circ} \mathrm{C}$ and

120 holding for $1 \mathrm{~h}$, then cooling to $40.0 \pm 0.5^{\circ} \mathrm{C}$. Gelatin-pectin mixed hydrogels (gel-pec) were

121 produced by separately preparing a gelatin (32.5 wt.-\%)/glycerol (17.15 wt.-\%) water

122 solution, according to the previous procedure, and dissolving $2.56 \mathrm{wt} .-\%$ pectin in hot water

$123\left(90^{\circ} \mathrm{C}\right)$ with vigorous stirring $(1000 \mathrm{rpm})$. Then, an aliquot of the pectin solution $(40.0 \pm 0.5$

$124{ }^{\circ} \mathrm{C}$, native $\left.\mathrm{pH} \sim 4.2\right)$ was added to a same aliquot of the gelatin-glycerol water solution (40.0

$125 \pm 0.5{ }^{\circ} \mathrm{C}$ ), to give a final concentration of 13 wt.- $\%$ gelatin, 7 wt.- $\%$ glycerol and 1 wt.- $\%$

126 pectin. After complete interdispersion of the solutions, the temperature was decreased to 37.0

$127 \pm 0.5{ }^{\circ} \mathrm{C}$. At this temperature, glutaraldehyde $(0.3$ wt. $-\%)$ was then added to an aliquot of this

128 solution under thorough and continuous mixing (550 rpm) to crosslink the gelatin and form

129 the third hydrogel (cross).

130 Once formed, all hydrogels were degassed and spread over the bottom of either Petri

131 dishes (100 mm diameter) or polycarbonate rectangular templates (300 mm length $\times 150 \mathrm{~mm}$

132 width), depending on the specific successive analysis. Final films were obtained after

133 evaporation of water in a vacuum oven (Model 282, Fisher Scientific, Pittsburgh, PA) at 40.0

$134 \pm 0.5^{\circ} \mathrm{C}$ for $24 \mathrm{hrs}$. Crosslinked films were additionally washed several times with Milli-Q

135 water and then air-dried at room temperature for 24 hours. The thicknesses of the final

136 structures, measured to the nearest $0.001 \mathrm{~mm}$ with a micrometer (Dialmatic DDI030M,

137 Bowers Metrology, Bradford, UK) at 10 different locations selected randomly, was $100 \pm 5$

$138 \mu \mathrm{m}$. 


\subsubsection{Scanning electron microscopy analysis (SEM)}

Strips of dry film (5mm wide x $30 \mathrm{~mm}$ long) were immersed in $20 \mathrm{ml}$ aliquots of 80 wt.- $\%$ ethanol water solution, with several changes. Then $80 \%$ ethanol was decanted and replaced with several changes of absolute ethanol. Next, strips were removed from absolute ethanol, quickly blotted dry and immersed in liquid Nitrogen for 5 minutes. The frozen strips were cross fractured manually using cold tweezers and the fractured pieces were thawed in absolute ethanol. Finally, the fragments were critical point dried from liquid $\mathrm{CO}_{2}$, and the dried fragments were glued to specimen stubs with Duco cement (ITW Performance Polymers, Riviera, FL), sputter-coated with a thin layer of gold and examined with a Quanta 200 FEG scanning electron microscope (FEI Co., Inc., Hillsboro, OR), operated in the high vacuum, secondary electron imaging mode. At least 10 images were collected for each sample.

\subsubsection{Atomic force microscopy analysis (AFM)}

Small areas (3-4 $\mathrm{mm}$ square) of films were cut with surgical scissors, glued to a magnetic sample disk with a carbon adhesive tab (Electron Microscopy Sciences, Hatfield, PA) and mounted on the scanner tube of a Multimode Scanning Probe microscope with a Nanoscope IIIa controller, operated as an atomic force microscope in Tapping mode (Veeco Instruments, Santa Barbara, CA). Small (1.0 and 2.5 micrometer square) areas of the samples were then scanned with the AFM operating in intermittent contact mode using tapping mode etched silicon probes (TESP). The spring constants for these probes were $20-100 \mathrm{~N} \mathrm{~m}^{-1}$ and the nominal tip radius of curvature was $5-10 \mathrm{~nm}$. The cantilever controls, namely drive frequency, amplitude, gains, and amplitude set point ratio $\left(r_{\mathrm{sp}}\right)$ were adjusted to give height and phase-shift images with the clearest image details. 


\subsubsection{Large deformation analysis}

Stress-strain curves of $110 \mathrm{~mm}$ x $20 \mathrm{~mm}$ strips of films equilibrated at $50 \%$ relative humidity conditions were collected according to the ASTM procedure (ASTM standard method D882-97) in a typical tensile test using a TAXT2 Stable Micro System texture analyzer (SMS, Surrey, UK) equipped with a $25 \mathrm{~kg}$ cell load. The initial grip separation was $100 \mathrm{~mm}$, and the cross-head speed was set at $0.85 \mathrm{~mm} \mathrm{~s}^{-1}$. Elastic modulus (or Young's modulus, $\mathrm{MPa})$, tensile strength (MPa), and strain at break (\%) were automatically calculated by the software Texture Expert version 1.15 (SMS, Surrey, UK). Each type of film was tested by at least ten replicates.

\subsubsection{Thermal properties (DSC)}

Thermal properties were measured by differential scanning calorimetry (DSC) analysis, using a DSC 823 (Mettler Toledo, Columbus, $\mathrm{OH}$ ) with a quench-cooling accessory. Aliquots of approximately $10 \mathrm{mg}$ samples previously conditioned $\left(23^{\circ} \mathrm{C}, 50 \% \mathrm{RH}\right.$ for 2 weeks) were placed in hermetically-sealed aluminium pans to prevent moisture loss during analyses and then heated at $10{ }^{\circ} \mathrm{C} \min ^{-1}$ from $5{ }^{\circ} \mathrm{C}$ to $110{ }^{\circ} \mathrm{C}$ in an inert environment $\left(100 \mathrm{ml} \mathrm{min}^{-1} \mathrm{~N}_{2}\right)$. The first scan was immediately followed by quick cooling to $5{ }^{\circ} \mathrm{C}$ at a rate of $40{ }^{\circ} \mathrm{C} \mathrm{min}{ }^{-1}$ using liquid nitrogen and the second scan was then run. Before taking the measurements, the instrument was calibrated with an indium standard $\left(\Delta \mathrm{H}\right.$ of $28.4 \mathrm{~J} \mathrm{~g}^{-1}$ and $\mathrm{Tm}$ of $\left.156.6{ }^{\circ} \mathrm{C}\right)$. The glass transition temperature $\left(\mathrm{T}_{\mathrm{g}}\right)$ of all the samples was determined as the point of inflexion in the base line (second scan) caused by the discontinuity of specific heat capacity of the sample. The helix-coil transition temperature, $\mathrm{T}_{\mathrm{m}}$, also called interchangeable melting or denaturation temperature (Arvanitoyannis, Nakayama, \& Aiba, 1998), was measured as the temperature of the endothermic peak (first scan). The value of helix-coil transition enthalpy $(\Delta H)$ was assumed derived from the amount of renaturated gelatin during the sol-gel process (Dai, 
191 Chen, \& Liu, 2005), and was normalized to the sample weight determined immediately before

192 each measurement. Tg, Tm, and $\Delta \mathrm{H}$ were calculated by the software $\mathrm{STAR}^{\mathrm{e}}$ version 9.0

193 (Mettler Toledo, Columbus, OH).

194

195

196

197

198

199

200

201

202

203

204

205

206

207

\subsubsection{Dynamic mechanical analysis (DMA)}

Dynamic mechanical properties (storage modulus - E', and loss modulus - E'’) were determined in the tensile mode using a Rheometric Scientific RSA II Solids Analyzer (Rheometric Scientific, Piscataway, NJ) equipped with Orchestrator 6.5.7 software. Samples (38.1 $\mathrm{mm}$ long and 5-7 mm wide) were analyzed as described previously (Coffin, \& Fishman, 1994), using a temperature ramp from -50 to $+150^{\circ} \mathrm{C}$ at a heating rate of $10^{\circ} \mathrm{C} \mathrm{min}^{-1}$.

\subsubsection{Swelling behaviour}

To evaluate the water sorption resistance of the gelatin-based films, square pieces of dry samples were weighed $\left(\mathrm{W}_{\mathrm{i}}\right)$ and then immersed in distilled water at $30^{\circ} \mathrm{C}$ with shaking $(100$ rpm) for up to 25 hours. Swollen gels were removed from water periodically, blotted dry, and weighed $\left(\mathrm{W}_{\mathrm{f}}\right)$ to track sorption kinetics. The swelling index $(\mathrm{SI})$ was determined as described by others (Myung et al., 2008):

$\operatorname{SI}(\%)=\left[\left(\mathrm{W}_{\mathrm{f}}-\mathrm{W}_{\mathrm{i}}\right) / \mathrm{W}_{\mathrm{i}}\right] \times 100$

Five replicates were analyzed for each time point.

\subsubsection{Statistical analysis}

Statistical significance of differences in films properties and behaviours was determined from one-way ANOVA using Statgraphics Plus 4.0 software (STSC, Rockville, USA). The mean values, where appropriate, were separated by least significant difference multiple range test at $p \leq 0.05$. 


\section{Results and Discussion}

216 3.1. Structural analysis - Microscopy experiments

\subsubsection{SEM analysis}

More global organization was revealed by scanning electron microscopy. SEM images of frozen-fractured surfaces of gelatin (gel) films showed rough, overlapping layers (10000x magnification, Figure 1, left) at the fracture faces, composed of ropelike aggregates in a relatively ordered arrangement (25000x magnification, Figure 1, right). With the inclusion of pectin, the pattern of topographical features in the fracture faces appears more irregular (Figure 2, left), whereas higher magnification reveals regions with larger features (Figure 2, right). Since the magnification of Figures 1 and 2 are the same, a comparison demonstrates that gelatin is represented by the smaller regions in Figure 2, whereas it is more difficult to ascertain whether the structures that stand out in Figure 2 (the larger domains) are either (1) the pectin, (2) gelatin with increased self-association forced by exclusion from areas with pectin, or (3) gelatin-pectin intertwined complexes. However, supported by previous results (Gilsenan et al., 2003), we believe that the large patches are produced by gelatin-pectin complexes. This is because, under the experimental conditions (i.e., $\mathrm{pH}=4.5$ ), the gelatin backbone exhibits an overall positive charge, whereas the carboxyl group along the pectin skeleton are indeed carboxylates. Therefore, due to the addition of pectin, positively charged gelatin and negatively charged pectin would be expected to interact through electrostatic forces between $\mathrm{NH}_{3}{ }^{+}$and $\mathrm{COO}^{-}$groups. Addition of $0.3 \mathrm{wt} \%$ glutaraldehyde produced more evident heterogeneity in the microstructure. In particular, the lumpy structures may represent large groups of polymers (triangles in Figure 3) with a separate phase entrapped within (arrows in Figure 3). The largest domains probably represent glutaraldehyde-mediated protein-polysaccharide interactions (Nikolova et al., 2005). Lately, it has been proved that these aggregates are the evidence of a new crosslinking mechanism of gelatin mediated by 
240 glutaraldehyde. Unlike the established reaction between the carbonyl group of glutaraldehyde 241 and the unprotonated $\varepsilon$-amino groups of lysine to form Schiff bases, it has been proposed that 242 at acidic $\mathrm{pH}$ the crosslinking occurs prevalently between the aldehyde groups of the 243 crosslinker and hydroxyl groups of hydroxyproline and hydroxylysine of gelatin (91 and 6.4 244 residues per 1000 residues in type A gelatin, respectively) to form hemiacetals (Farris, Song, 245 \& Huang, 2010), according to the mechanism shown in Figure 4. As we pointed out, it is 246 likely that under the experimental conditions, unprotonated amino groups of gelatin and 247 carbonyl groups of the crosslinker contribute to the formation of new bridges only to a minor 248 extent. The resulting structure can be seen as a tightly packed three-dimensional network 249 composed of a primary entangled phase of chemically-crosslinked gelatin, arranged into 250 fibers that connect and encase a secondary component - pectin, linked to gelatin by ionic 251 interactions. Together these components formed what has been defined as a 'permanent 252 polyion-complex gel' (Farris et al. 2009b).

\subsubsection{AFM analysis}

In order to understand the structures formed during the gelation process more thoroughly, interfacial gelatin and gelatin-pectin films formed at the air-water interface were also studied using atomic force microscopy (AFM). The AFM 'height' image of films obtained from glycerol-plasticized gelatin (Figure 5, left) well correlates with the molecular network highlighted by Morris and co-workers through the same technique (Morris, Kirby, \& 259 Gunning, 1999). Gelatin molecules assemble into aggregates containing short segments of 260 dimensions comparable to those expected for collagen triple helices. The image shows small aggregates typically $200-400 \mathrm{~nm}$ in length and about $6 \mathrm{~nm}$ in height. The aggregates appear to be clusters of molecules presumably linked by intermolecular triple helix formation. It has helical junction zones (Mackie, Gunning, Ridout, \& Morris, 1998). Addition of pectin led to a 
completely different scenario. Figure 6 (left) revealed that the above-mentioned gelatin 266 clusters disappear giving way to a more homogeneous structure. In particular, it seems that pectin hindered the possibility for gelatin to recover the original triple helix conformation, while promoting the formation of a binary network in which gelatin and pectin are supposed to interact through ionic interactions, as mentioned above. The 'phase' image indeed confirmed the compatibility between the two components, which generated a highly smooth surface, especially in comparison with the 'phase' image drawn from gelatin films (Figure 5, right). As already pointed out (Dautzenberg \& Jaeger, 2002), the effect of charge density (which can be manipulated by the $\mathrm{pH}$ of the system) on the stability of polyelectrolyte complexes must be kept under control. Therefore, also in our system the charge balance 275 (which is controlled by the polyelectrolytes ratio in the mixed dispersion) between gelatin and 276 pectin plays a pivotal role in order to have a uniformly distributed network. Combining 277 gelatin and pectin in different stoichiometries alters the positive/negative charge balance and hence the extent of associations between these two polyions (Farris et al., 2009b). Our results demonstrate that combining 13 wt.- $\%$ of type A 250 Bloom gelatin and 1 wt.- $\%$ low methoxyl 280 pectin $(\mathrm{DE}=7)$ at native $\mathrm{pH}(\sim 4.5)$ generates a physical polyion complex composed of a 281 main gelatin network augmented by additional associations through ionic interactions with 282 pectin, with no evidence of either large domains from self-aggregations or precipitation upon 283 complex formation. However, when the molar excess of one component is too great, 284 segregative interactions occur and the system evolves into two co-existing phases (Gilsenan et 285 al., 2003). This phenomenon is shown in Figure 7 (left) where gelatin domains (the darker 286 regions) are separated from the pectin molecules (the bright 'scrapes'), which in turn are 287 associated through homotypic junctions analogously to a single-component gel. The final result is a rougher topography (Figure 7, right). Finally, AFM images of crosslinked samples 289 are consistent with the results from the SEM analysis. The morphology of the final structure is 290 strongly influenced by the addition of glutaraldehyde, which induced the formation of new 
291 bonds between gelatin molecules especially at intermolecular level (Farris et al., 2010), 292 yielding a more pronounced web-like conformation, with pectin molecules forming part of it 293 (Figure 8, left). At the same time, due to these new domains, the surface topography appears 294 rough and noisy (Figure 8, right).

\subsubsection{Large deformation analysis}

As shown by Figure 9, the mean stress-strain curves for the three types of films differ from each other, as also confirmed by the results reported in Table 1. The elastic modulus values were calculated for all specimens from the slope of the linear climbing tract of the stress-strain plot within the fixed strain region $0.5 \%-1.0 \%$, as visualized in the magnification embedded in Figure 9. The elastic modulus values recorded for the (gel) samples are approximately two and one and half times higher than those obtained for (gel-pec) and (cross) samples, respectively (Table 1). This can be ascribed to the lower degree of crystallinity of (gel-pec) and (cross) samples. Both interactions governed by electrostatic forces and covalent bonds reduced the crystallinity degree of the gelatin main network because close packing of molecules is prevented, thus resulting in lower stiffness of the polymer (Andersson, 2008).

308 Since the slope of the first rising tract of a stress-strain curve gives a measure of the material's stiffness, which is normally assumed to be an indication of the degree of crystallinity (Selke,

310 Culter, \& Hernandez, 2004), it can be concluded that the gelatin samples had a higher degree 311 of crystallinity than the other samples. Our results agree with those obtained by Thomazine, 312 Carvalho and Sobral (2005), where gelatin films plasticized with glycerol (55 wt\% of gelatin 313 content) had an elastic modulus mean value of $0.41 \pm 0.11 \mathrm{MPa}$. Our results also show how 314 the physical hydrogel solution made by the negatively charged pectin and the positively charged gelatin led to films with tensile strength and elongation at break respectively $26 \%$ and 
$24 \%$ higher than samples obtained from only gelatin. Addition of glutaraldheyde made it

317 possible to obtain films with still further improved mechanical properties. Presumably, 318 uneven crosslinking occurred during films preparation led to development of new zones in 319 which typical 'irreversible' features (new covalent bonds at both intramolecular and 320 intermolecular level) are dominant compared to the still present 'reversible' domains, in 321 which interactions within and between molecules are mainly governed by weak forces 322 (hydrophobic associations, ionic interactions, or hydrogen bonding) and/or physical entanglement. As can be seen from Table 1, crosslinking increased both tensile strength and elongation at break, though the latter was not statistically different from samples obtained from the gelatin-pectin physical hydrogel solution. In order to substantiate the positive role of the electrostatic interactions between gelatin and pectin, we also performed the tensile test on samples obtained from only gelatin crosslinked with glutaraldehyde. These films had elastic modulus, tensile strength and elongation at break values of $0.22 \pm 0.05 \mathrm{MPa}, 14.01 \pm 2.36$ $\mathrm{MPa}$ and $149.3 \pm 8.09 \%$, respectively. These results suggest that the best performance of 330 crosslinked gelatin-pectin films can be presumably ascribed to the additional effect due to the 331 electrostatic interactions between the oppositely charged functional groups of the two 332 biomacromolecules.

\subsubsection{Thermal properties (DSC)}

Figure 10 shows the differential scanning calorimetry (DSC) traces obtained from the

335 first scan of pure gelatin, gelatin-pectin, and gelatin-pectin films crosslinked using 336 glutaraldehyde. All traces display the classical thermal behaviour of gelatin samples, with the 337 first drop of the curve related to the glass transition, followed by an endothermic peak 338 associated with the helix to coil transition. In the second heating scan, only the glass transition 339 is evident (a typical heating-cooling-heating DSC trace for non-crosslinked glycerol340 plasticized gelatin samples equilibrated at 50\% relative humidity is displayed in Figure 11). 
341 This is due to the disruption of the microcrystalline domains during the first heating cycle.

342 From Figure 10 it can be observed how (gel) and (gel-pec) samples provided very similar 343 traces, contrary to (cross) samples, which led to a curve with an anticipated and less 344 pronounced peak. More specifically, (gel) samples had $\mathrm{T}_{\mathrm{g}}=50.53 \pm 0.6{ }^{\circ} \mathrm{C}, \mathrm{T}_{\mathrm{m}}=59.45 \pm 0.3$ $345{ }^{\circ} \mathrm{C}$, and $\Delta \mathrm{H}=10.9 \pm 0.3 \mathrm{~J} \mathrm{~g}^{-1}$. (Gel-pec) samples were characterized by $\mathrm{T}_{\mathrm{g}}=51.23 \pm 0.7^{\circ} \mathrm{C}$, $346 \mathrm{~T}_{\mathrm{m}}=59.23 \pm 0.2^{\circ} \mathrm{C}$, and $\Delta \mathrm{H}=9.4 \pm 0.5 \mathrm{~J} \mathrm{~g}^{-1}$. Finally, crosslinked samples yielded $\mathrm{T}_{\mathrm{g}}=44.78$ $347 \pm 0.6{ }^{\circ} \mathrm{C}, \mathrm{T}_{\mathrm{m}}=50.37 \pm 0.4{ }^{\circ} \mathrm{C}$, and $\Delta \mathrm{H}=3.2 \pm 0.4 \mathrm{~J} \mathrm{~g}^{-1}$. The overall lower values for 348 crosslinked samples can be explained by taking into consideration two different effects.

349 Firstly, the appearance of the endothermic peak in a DSC curve is normally due to the 350 breakage of hydrogen bonds. Conversely, when a crosslinked network structure is involved, a 351 small or negligible endothermic peak is expected. Therefore, the decrease in the helix-to-coil 352 enthalpy values $(\Delta \mathrm{H})$ when using glutaraldehyde has to be attributed to an increase in the 353 extent of crosslinked network formation which breaks exothermically. Secondly, the 354 simultaneous lower $\mathrm{T}_{\mathrm{g}}$ of (cross) samples can be explained by hypothesizing an 'inhibition 355 effect' exerted by the crosslinker, which prevented the recovery of the structurally-ordered 356 microcrystalline domains (the so called microcrystallites), to the advantage of a more 357 amorphous final molecular structure.

\subsubsection{Dynamic mechanical analysis (DMA)}

A dynamic mechanical analysis was performed on selected samples obtained from

360 hydrogel solutions containing only gelatin, gelatin and pectin, and gelatin and pectin with 361 glutaraldehyde. The storage modulus curves (E') are shown in Figure 12a, while the loss 362 modulus curves (E") are shown in Figure 12b. Both the storage modulus and loss modulus 363 curves for the gelatin-only samples had significantly lower values than did the curves for the 364 other two samples throughout the entire temperature range studied. Two transitions were 365 present in all three samples. The first was a peak in the loss modulus curve centered at 
approximately $-56^{\circ} \mathrm{C}$. This is likely due to a glass to rubber transition $\left(\mathrm{T}_{\mathrm{g}}\right)$ associated with soft

367 blocks containing mainly R-amino acids. A second peak near $50^{\circ} \mathrm{C}$ may be related to a second 368 glass transition of rigid gelatin blocks composed of sequences predominately made up of the 369 amino acids proline and hydroxyproline (Chiellini, Cinelli, Grillo Fernandes, El-Refaie, \& 370 Lazzeri, 2001). The addition of pectin to the gelatin increased both the storage modulus and 371 loss modulus of the material. Above $-60^{\circ} \mathrm{C}$, crosslinking had little or no effect on mechanical 372 properties, although a very slight decrease in modulus there may have resulted below this temperature. The results of the DMA analyses show some confirmation of what was

374 previously shown in the DSC experiment. In particular, there are no changes in thermal stability when the gelatin hydrogel is modified by the addition of pectin, and then further modified by reaction with glutaraldehyde.

\subsubsection{Swelling behaviour}

Results from swelling experiments are summarized in Figure 13. Curves obtained by plotting the swelling index versus time indicate how water absorption was markedly higher for (gel) samples, whereas differences between (gel-pec) and (cross) samples were negligible up to 120 minutes. In addition, (gel) samples gained water throughout the experiment, 382 whereas both uncrosslinked and crosslinked gelatin-pectin films reached a quite stable 383 equilibrium swelling level after approximately 240 minutes. Moreover, the experiments were stopped after 25 hours due to the impossibility of taking gelatin samples out of the stirring jars because of their complete disintegration. Conversely, it was still possible to handle (gel-pec) and (cross) samples even after 25 hours. It is noticeable that at that time, (gel-pec) pieces of

387 samples cannot sustain any kind of stress without failure, while it was possible to stretch 388 (cross) samples without rupture, somehow confirming the reversible nature of the (gel-pec) samples and the permanent feature of crosslinked samples. These results confirm the benefits

390 arising from the addition of pectin first and crosslinker later. Exploiting electrostatic 
391 interactions between the two biomacromolecules made it possible to achieve a dramatic 392 decrease in the ultimate swelling ratio (300\% versus $1950 \%$ of gelatin samples). After all, 393 electrostatic self-assembly is a widely established route to generate supramolecular structures 394 with enhanced properties (Grohn, 2008). Presumably, electrostatic interactions between 395 gelatin and pectin hinder penetration of water molecules, which will take a longer time to 396 compete for the same hydrophilic sites along the molecular skeleton of the two biomolecules. 397 Inducing new permanent bonds through glutaraldehyde further enhanced this trend. Indeed, 398 films crosslinked with only 0.3 wt.-\% glutaraldehyde swelled approximately ten times less 399 than uncrosslinked gelatin samples (215\% versus $1950 \%)$ after 25 hours, indicating how the 400 irreversible attribute of the hydrogel solution initially used to produce the films provided a 401 greater water resistance. As also suggested by AFM images (Figure 7), it is likely that the 402 addition of the crosslinker led to a heterogeneous molecular arrangement, due to the presence 403 of domains with an uneven degree of crosslinking, which determines the simultaneous 404 presence of crosslinked undissolvable patches together with zones in which the typical 405 'reversible' features are still dominant. These features are responsible for the occurred 406 swelling, which in turn strictly depends on the extent of the crosslinking. As a consequence, a 407 slightly higher concentration of glutaraldehyde within a certain limit should afford additional 408 resistance to swelling, without jeopardizing the film-making process (Bigi, Cojazzi, 409 Panzavolta, Rubini, \& Roveri, 2001).

410 Finally, the role of glycerol in the ultimate water resistance property of the obtained films 411 should not be underestimated. Glycerol was here used as a plasticizer, which provides films 412 with improved mechanical properties especially in terms of flexibility. However, both the 413 small size and polar nature of this molecule influence the physical structure of the 414 biopolymers (increasing the free volume between adjacent molecules) as well as their affinity 415 to water. Therefore, the tendency of water sorption becomes even more significant. 
416 Depending on the specific application, either replacing glycerol with other molecules or

417 removing it from the starting formulation may provide desirable benefits.

\section{Conclusions}

The results arising from our investigation showed the importance of developing a proper

421 hydrogel solution for producing ultimate films with improved overall properties. In particular, 422 microscopy analyses corroborate the hypothesis that the addition of pectin to gelatin 423 according to a well defined ratio leads to a composite network in which the protein and the 424 polysaccharide interact with each other through electrostatic forces. It afforded structures with improved mechanical and water resistance properties, which could be profitably exploited for manufacturing special materials, in order to replace some of the commercial solutions currently used. In addition, crosslinking mediated by glutaraldehyde promoted the formation of covalent bonds between gelatin molecules, without interfering with the previously mentioned physical interactions, and allowing the formation of an interconnected gelatin web with the pectin network dispersed inside. Physical characterization of gelatin-based films indirectly supports our findings. The molecular arrangement we propose indeed justifies the increased performance of the obtained structures, which demonstrates the capability of withstand high loads simultaneously to a great elongation, and good water resistance as well

434 (films swelled, but never dissolved nor disintegrated).

Acknowledgements: we are thankful to Dr. D. Coffin and G. Bao for technical and scientific assistance. 


\section{References}

442 Andersson, C. (2008). New ways to enhance the functionality of paperboard by surface 443 treatment - a review. Packaging Technology and Science, 21 (6), 339-373.

444 Arvanitoyannis, I.S., Nakayama, A., \& Aiba, S. (1998). Chitosan and gelatin based edible 445 films: state diagrams, mechanical and permeation properties. Carbohydrate Polymers, 37 (4), $446 \quad 371-382$.

447 ASTM 1997. Designation D 882-97: Standard test method for tensile properties of thin plastic 448 sheeting. Annual Book of ASTM Standards; American Society for Testing and Materials: 449 Philadelphia, 1997, p 159.

450 Bigi, A., Cojazzi, G., Panzavolta, S., Rubini, K., \& Roveri, N. (2001). Mechanical and 451 thermal properties of gelatin films at different degrees of glutaraldehyde crosslinking. 452 Biomaterials, $22(8), 763-768$.

453 Chiellini, E., Cinelli, P., Grillo Fernandes, E., El-Refaie, S.K., \& Lazzeri, A. (2001). Gelatin454 based blends and composites. Morphological and thermal mechanical characterization. 455 Biomacromolecules, 2 (3), 806-811.

456 Coffin, D.R., \& Fishman, M.L. (1994). Physical and mechanical properties of highly 457 plasticized pectin/starch films. Journal of Applied Polymer Science, 54 (9), 1311-1320.

458 Dai, C.A., Chen, Y.F., \& Liu, M.W. (2006). Thermal properties measurements of renatured 459 gelatin using conventional and temperature modulated differential scanning calorimetry. 460 Journal of Applied Polymer Science, 99 (4), 1795-1801.

461 Dautzenberg, H., \& Jaeger, W. (2002). Effect of charge density on the formation and salt 462 stability of polyelectrolyte complexes. Macromolecular Chemistry and Physics, 203 (14), $463 \quad 2095-2102$. 
464 De Kruif, G., \& Tuinier, R. (2001). Polysaccharide protein interactions. Food Hydrocolloids, $46515(4-6), 555-563$.

466 Farris, S., Introzzi, L., \& Piergiovanni, L. (2009a). Evaluation of a bio-coating as a solution to 467 improve barrier, friction and optical properties of plastic films. Packaging Technology and 468 Science, $22(2), 69-83$.

469 Farris, S., Schaich, K.M., Liu, L., Piergiovanni, L., \& Yam, K.L. (2009b). Development of 470 polyion-complex hydrogels as an alternative approach for the production of bio-based 471 polymers for food packaging applications: a review. Trends in Food Science and Technology, $472 \quad 20(8), 316-332$.

473 Farris, S., Song, J., \& Huang, Q. (2010). Alternative reaction mechanism for the cross-linking 474 of gelatin with glutaraldehyde. Journal of Agricultural and Food Chemistry, 58 (2), 9984751003.

476 Ghaffari, A., Navaee, K., Oskoui, M., Bayati, K., \& Rafiee-Tehrani, M. (2007). Preparation 477 and characterization of free mixed-film of pectin/chitosan/Eudragit ${ }^{\circledR}$ RS intended for 478 sigmoidal drug delivery. European Journal of Pharmaceutics and Biopharmaceutics, 67 (1), $479 \quad 175-186$.

480 Giancone, T., Torrieri, E., Masi, P., \& Michon, C. (2009). Protein-polysaccharide 481 interactions: phase behaviour of pectin-soy flour mixture. Food Hydrocolloids, 23 (5), 1263 4821269.

483 Gilsenan, P.M., Richardson, R.K., \& Morris, E.R. (2003). Associative and segregative 484 interactions between gelatin and low-methoxy pectin: Part 1. Associative interactions in the 485 absence of $\mathrm{Ca}^{2+}$. Food Hydrocolloids, 17 (6), 723-737. 
Gong, J.P., Katsuyama, Y., Kurokawa, T., \& Osada, Y. (2003). Double-network hydrogels with extremely high mechanical strength. Advanced Materials, 15 (14), 1155-1158.

Gröhn, F. (2008). Electrostatic self-assembly as route to supramolecular structures. Macromolecular Chemistry and Physics, 209 (22), 2295-2301.

Hoare, T.R., \& Kohane, D.S. (2008). Hydrogels in drug delivery: progress and challenges. Polymer, 49 (8), 1993-2007.

Hoffman, A.S., Stayton, P.S., Press, O., Murthy, N., Lackey, C.A., Cheung, C., Black, F., Campbell, J., Fausto, N., Kyriakides, T.R., \& Bornstein, P. (2002). Design of smart polymers that can direct intracellular drug delivery. Polymers for Advanced Technologies, 13 (10), 992999.

Jaklenec, A., Wan, E., Murray, M.E., \& Mathiowitz, E. (2008). Novel scaffolds fabricated from protein-loaded microspheres for tissue engineering. Biomaterials, 29 (2), 185-192.

Jeong, B., Kim, S.W., \& Bae, Y.H. (2002). Thermosensitive sol-gel reversible hydrogels. Advanced Drug. Delivery Reviews, 54 (1), 37-51.

Khademhosseini, A., \& Langer, R. (2007). Microengineered hydrogels for tissue engineering. Biomaterials, 28 (34), 5087-5092.

Kurisawa, M., \& Yui, N. (1998). Gelatin/dextran intelligent hydrogels for drug delivery: dualstimuli-responsive degradation in relation to miscibility in interpenetrating polymer networks. Macromolecular Chemistry and Physics, 199 (8), 1547-1554.

Lai, J.Y., Lu, P.L., Chen, K.H., Tabata Y., \& Hsiue, G.H. (2006). Effect of charge and molecular weight on the functionality of gelatin carriers for corneal endothelial cell therapy. Biomacromolecules, 7 (6), 1836-1844. 
508 Lin, C.C., \& Metters, A.T. (2006). Hydrogels in controlled release formulations: network 509 design and mathematical modeling. Advanced Drug Delivery Reviews, 58 (12-13), 13795101408.

511 Liu, L., Liu, C.K., Fishman, M.L., \& Hicks, K.B. (2007). Composite films from pectin and 512 fish skin gelatin or soybean flour protein. Journal of Agricultural and Food Chemistry, 55 (6), $513 \quad 2349-2355$

514 Mackie, A.R., Gunning, A.P., Ridout, M.J., \& Morris, V.J. (1998). Gelation of gelatin 515 observation in the bulk and at the air-water interface. Biopolymers, 46 (4), 245-252.

516 Mohareb, E., \& Mittal, G.S. (2007). Formulation and process conditions for 517 biodegradable/edible soy-based packaging trays. Packaging Technology and Science, 20 (1), $518 \quad 1-15$.

519 Morris, V.J., Kirby, A.R., \& Gunning, A.P. (1999). Using atomic force microscopy to probe 520 food biopolymer functionality. Scanning, 21 (5), 287-292.

521 Mourya, V.K., \& Inamdar, N.N. (2008). Chitosan-modifications and applications: 522 Opportunities galore. Reactive and Functional Polymers, 68 (6), 1013-1051.

523 Myung, D., Waters, D., Wiseman, M., Duhamel, P.-E., Noolandi, J., Ta, C.N., \& Frank, C.W. 524 (2008). Progress in the development of interpenetrating polymer network hydrogels. Polymers 525 for Advanced Technologies, 19 (6), 647-657.

526 Nikolova, K., Panchev, I., \& Sainova, S. (2005). Optical characteristics of biopolymer films 527 from pectin and gelatin. Journal of Optoelectronics and Advanced Materials, 7 (3), 14395281444. 
Ong, S.Y., Wu, J., Moochhala, S.M., Tan, M.H., \& Lu, J. (2008). Development of a chitosanbased wound dressing with improved hemostatic and antimicrobial properties. Biomaterials, $29(32), 4323-4332$.

Ravi Kumar, M.N.V. (2000). A review of chitin and chitosan applications. Reactive and 533 Functional Polymers, 46 (1), 1-27.

534 Selke, S.E.M., Culter, J.D., \& Hernandez, R.J. (2004). Plastics Packaging: properties, 535 processing, applications, and regulations. Munich: Hanser Carl.

536 Skotak, M., Leonov, A. P., Larsen, G., Noriega, S., \& Subramanian, A. (2008). Biocompatible 537 and biodegradable ultrafine fibrillar scaffold materials for tissue engineering by facile grafting 538 of 1-lactide onto chitosan. Biomacromolecules, 9 (7), 1902-1908.

539 Tharanathan, R.N. (2003). Biodegradable films and composite coatings: past, present and 540 future. Trends in Food Science \& Technology, 14 (3), 71-78.

541 Thomazine, M., Carvalho, R.A., \& Sobral, J.A.P. (2005). Physical properties of gelatin films 542 plasticized by blends of glycerol and sorbitol. Journal of Food Science, 70 (3), 172-176.

543 Turgeon, S.L., Schmitt, C., \& Sanchez, C. (2007). Protein-polysaccharide complexes and 544 coacervates. Current Opinion in Colloid \& Interface Science, 12 (4-5), 166-178.

545 Weber, C.J., Haugaard, V., Festersen, R., \& Bertelsen, G. (2002). Production and applications 546 of biobased packaging materials for the food industry. Food Additives and Contaminants, 19 $547 \quad(s), 172-177$.

548 Wei, X., Sun, N., Wu, B., Yin, C., \& Wu, W. (2006). Sigmoidal release of indomethacin from 549 pectin matrix tablets: Effect of in situ crosslinking by calcium cations. International Journal 550 of Pharmaceutics, 318 (1-2), 132-138. 
551 Wooster, T.J., \& Augustin, M.A. (2007). Rheology of whey protein-dextran conjugate films

552 at the air/water interface. Food Hydrocolloids, 21 (7), 1072-1080.

553 Yao, C.H., Liu, B.S., Chang, C.J., Hsu, S.H., \& Chen, Y.S. (2004). Preparation of networks of

554 gelatin and genipin as degradable biomaterials. Materials Chemistry and Physics, 83 (2-3),

$555 \quad 204-208$.

556

557

558

559

560

561

562

563

564

565

566

567

568

569

570

571

572

573

574

575 
Figure captions

Figure 1. SEM photograph at two different magnifications (10000x - left, and 25000x - right) glycerol concentration: 7 wt.-\%; pH of the hydrogel solution: 4.5).

Figure 2. SEM photograph at two different magnifications (10000x - left, and 25000x - right) of frozen-fractured gelatin-pectin films plasticized with glycerol (gelatin concentration: 13 wt.-\%; pectin concentration: 1 wt.-\%; glycerol concentration: 7 wt.- $\%$; pH of the hydrogel solution: 4.4).

Figure 3. SEM photograph at two different magnifications (10000x - left, and 25000x - right) of frozen-fractured gelatin-pectin films plasticized with glycerol and crosslinked with glutaraldehyde (gelatin concentration: 13 wt.-\%; pectin concentration: 1 wt.-\%; glycerol concentration: 7 wt.-\%; glutaraldehyde concentration: $0.3 \%$; $\mathrm{pH}$ of the hydrogel solution: 4.4).

Figure 4. Mechanism for the crosslinking reaction of gelatin by glutaraldehyde at acidic $\mathrm{pH}$ 594 values.

Figure 5. Matching images of (A) height and (B) phase-shift of a gelatin films plasticized with glycerol (gelatin concentration: 14 wt.-\%; glycerol concentration: 7 wt.-\%; pH of the hydrogel solution: 4.5). The thin clefts or depressions in the surface of the sample in (A) correspond to gelatin triple helix aggregations. 
601 Figure 6. Matching images of (A) height and (B) phase-shift of a film made from a starting 602 glycerol-plasticized gelatin and pectin polyion complex hydrogel solution (gelatin 603 concentration: 13 wt.-\%; pectin concentration: 1 wt.-\%; glycerol concentration: 7 wt.-\%; pH 604 of the hydrogel solution: 4.4).

605

606 Figure 7. Matching images of (A) height and (B) phase-shift of a film made from a starting 607 glycerol-plasticized gelatin and pectin polyion complex hydrogel solution with an excess of 608 pectin (gelatin concentration: $10 \mathrm{wt.} \%$; pectin concentration: 4 wt.-\%; glycerol concentration: 6097 wt.-\%; pH of the hydrogel solution: 4.3). Dark domains are gelatin associations, whereas 610 the bright 'scrapes' are pectin clusters.

612 Figure 8. Matching images of (A) height and (B) phase-shift of a film made from a starting 613 glycerol-plasticized gelatin and pectin polyion complex hydrogel solution crosslinked with 614 glutaraldehyde (gelatin concentration: 13 wt.-\%; pectin concentration: 1 wt.-\%; glycerol 615 concentration: 7 wt.-\%; glutaraldehyde concentration: 0.3 wt.- $\%$; $\mathrm{pH}$ of the hydrogel solution: 616 4.4). Crosslinked patches appear as a dense web.

618 Figure 9. Tensile stress-strain curves of gelatin (14 wt.- $\%)$, gelatin-pectin (13 wt.-\% and 1 wt.-\%, respectively), and gelatin-pectin films crosslinked with glutaraldehyde (0.3 wt.-\%). All

620 films were plasticized with glycerol (7 wt.-\%). Experimental testing conditions: $23 \pm 0.5^{\circ} \mathrm{C}$, $62150 \pm 2.0 \%$ relative humidity.

623 Figure 10. DSC traces (first scan) of pure gelatin, gelatin-pectin, and gelatin-pectin 624 crosslinked films equilibrated at $23 \pm 0.5^{\circ} \mathrm{C}$ and $50 \pm 2.0 \%$ relative humidity before analysis. 
626 Figure 11. A typical heating-cooling-heating DSC trace for a glycerol-plasticized gelatin 627 film (gelatin: $14 \mathrm{wt}-\%$; glycerol: $7 \mathrm{wt}-\%$ ) equilibrated at 50\% relative humidity.

628

629 Figure 12. Storage modulus - E' (a) and loss mosulus - E" (b) of gelatin (14 wt.-\%), gelatin630 pectin (13 wt.-\% and 1 wt.-\%, respectively), and gelatin-pectin films crosslinked with 631 glutaraldehyde (0.3 wt.-\%). All films were plasticized with glycerol (7 wt.-\%).

632

633 Figure 13. Swelling index evolution of gelatin (14 wt.-\%), gelatin-pectin (13 wt.-\% and 1 wt.$634 \%$, respectively), and gelatin-pectin films crosslinked with glutaraldehyde (0.3 wt.-\%) up to 63525 hours immersion in distilled water $\left(30^{\circ} \mathrm{C}, 100 \mathrm{rpm}\right)$. All films were plasticized with 636 glycerol (7 wt.-\%). 

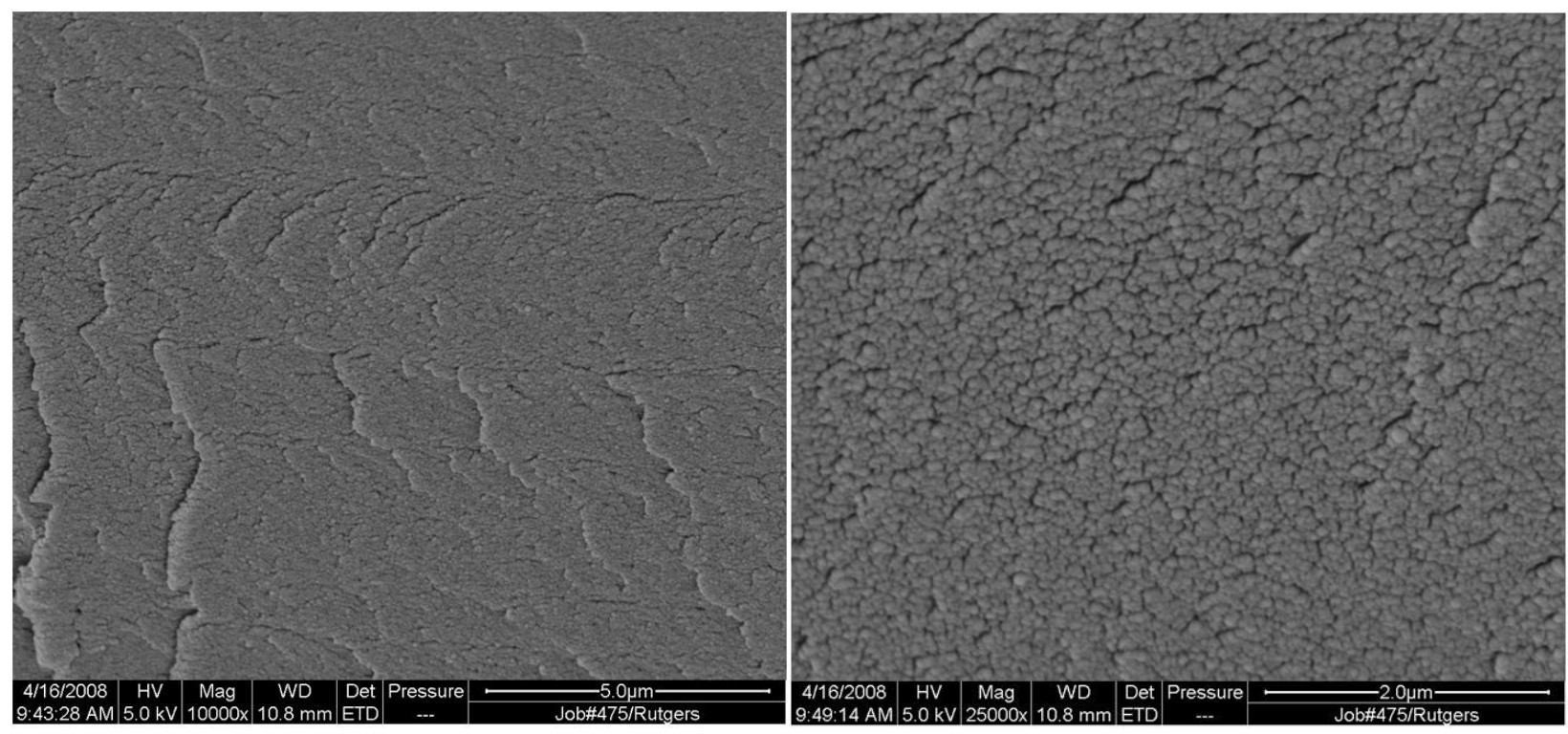

Figure 1 

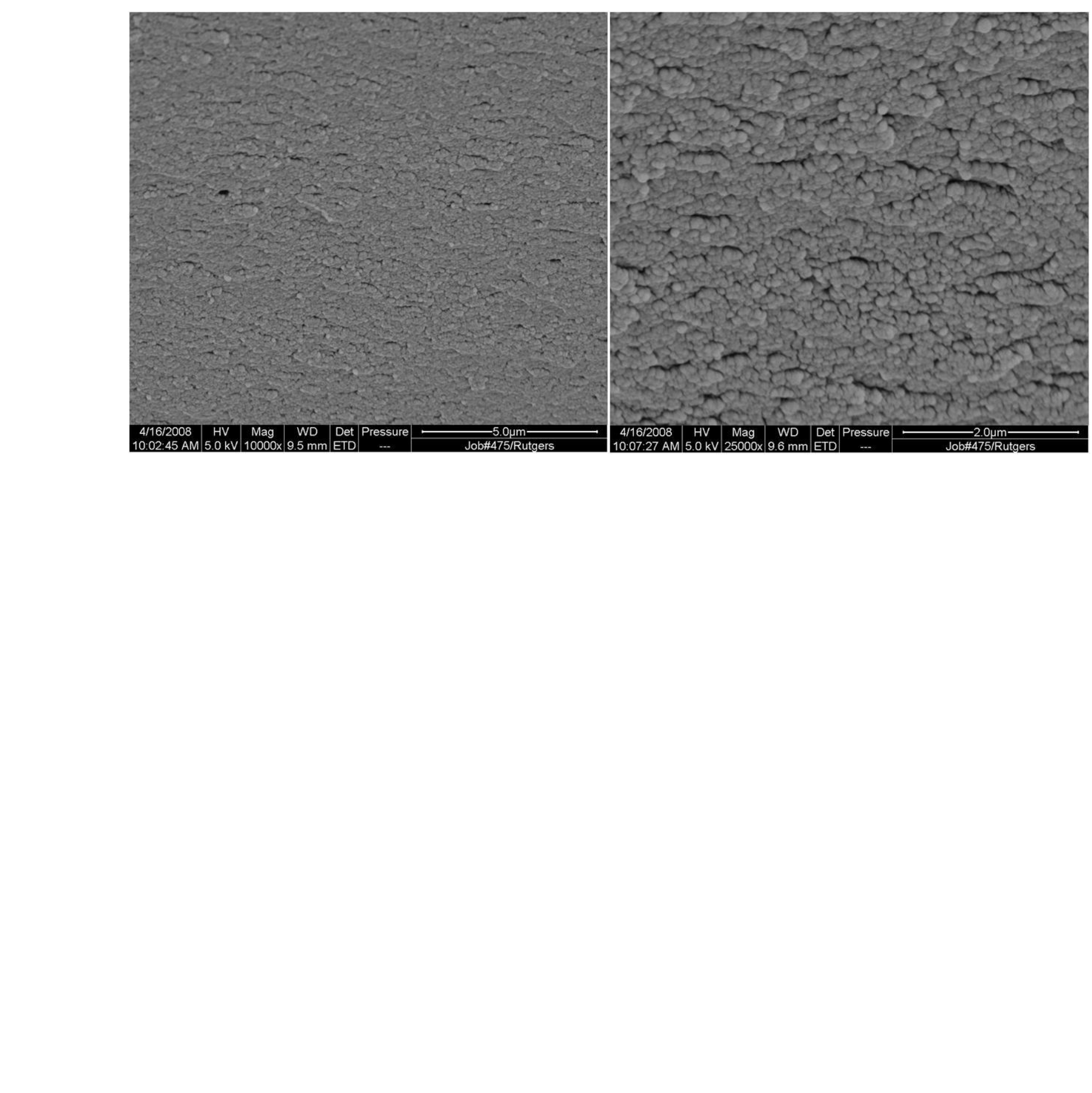

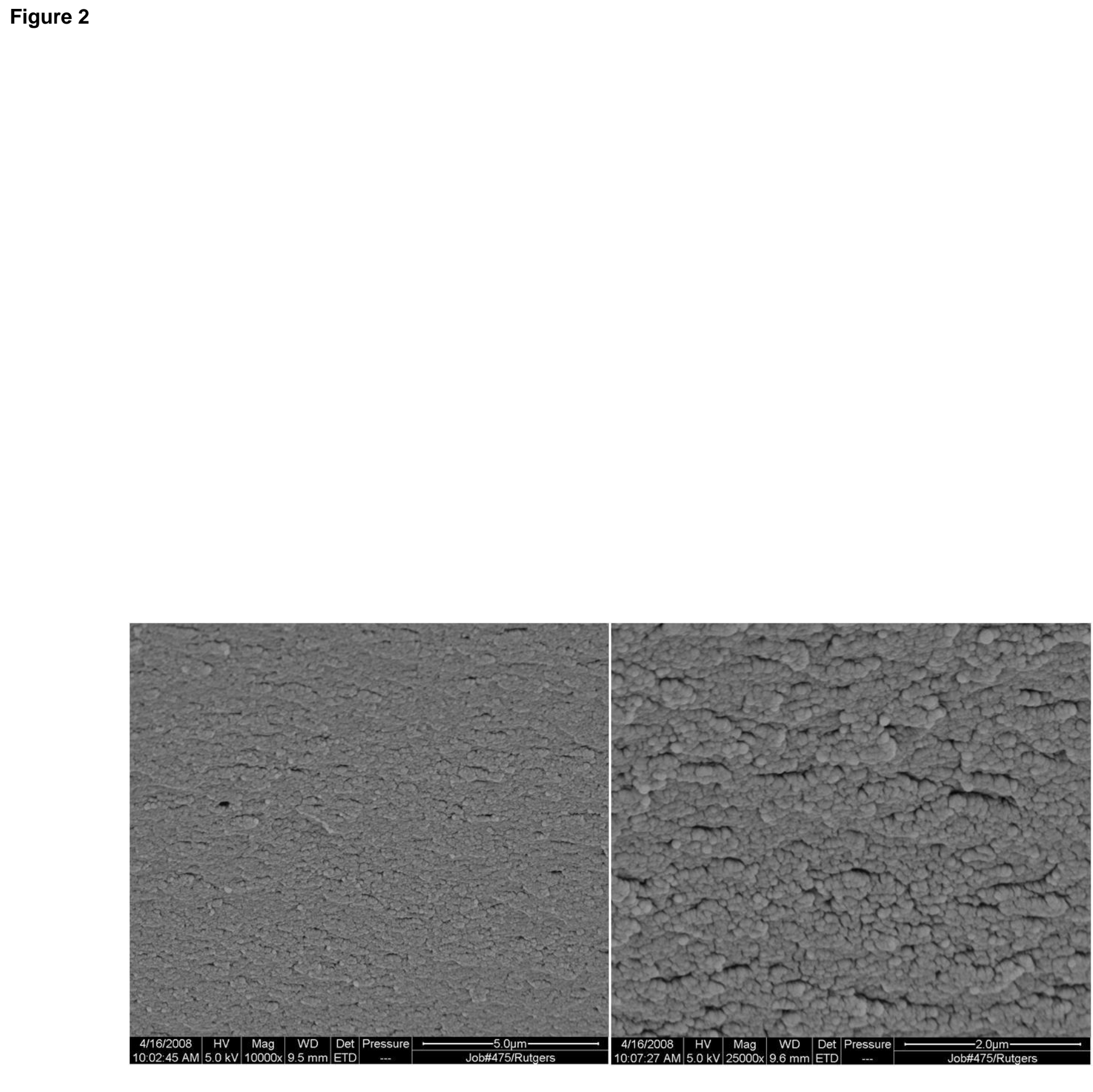

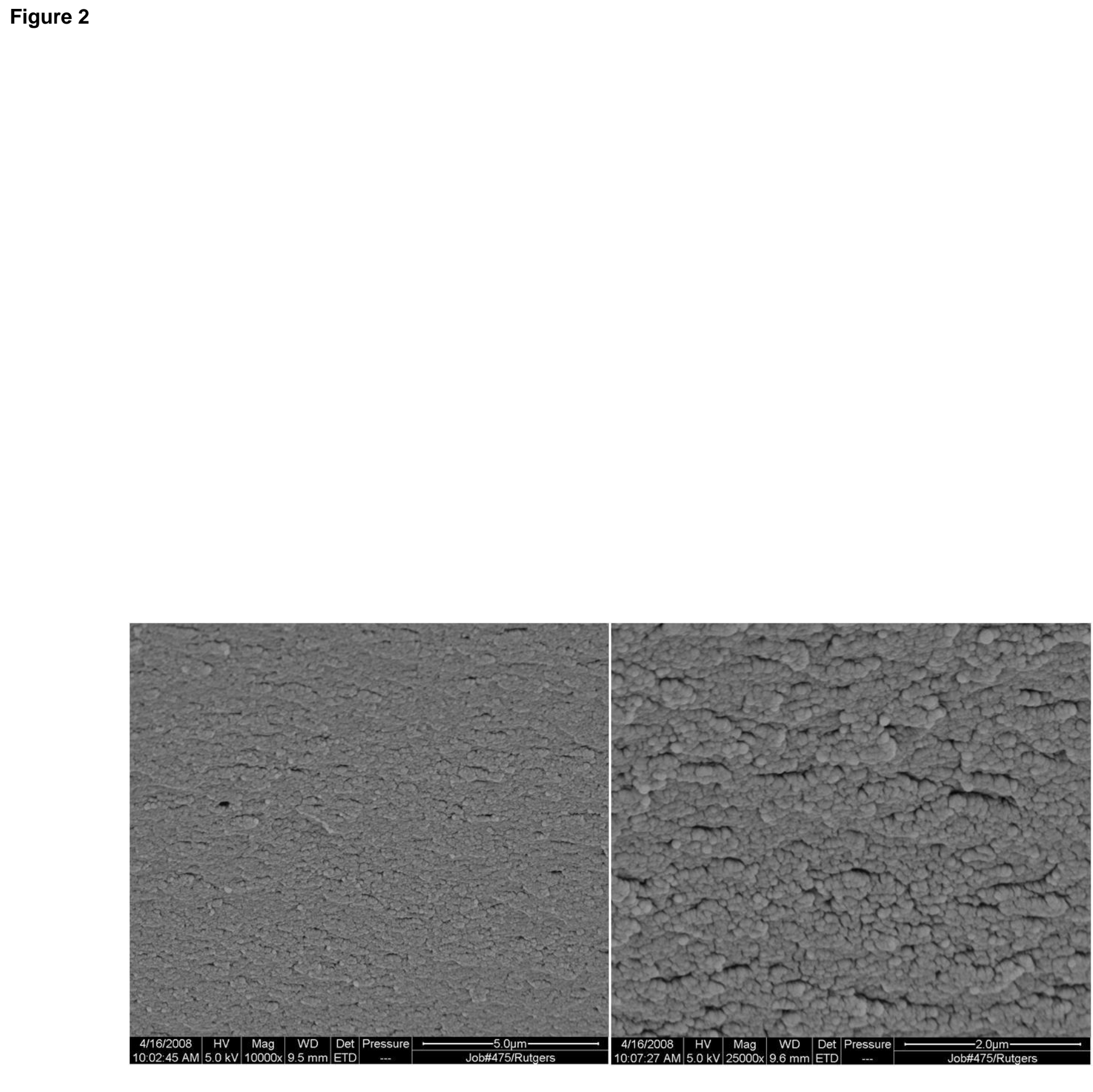

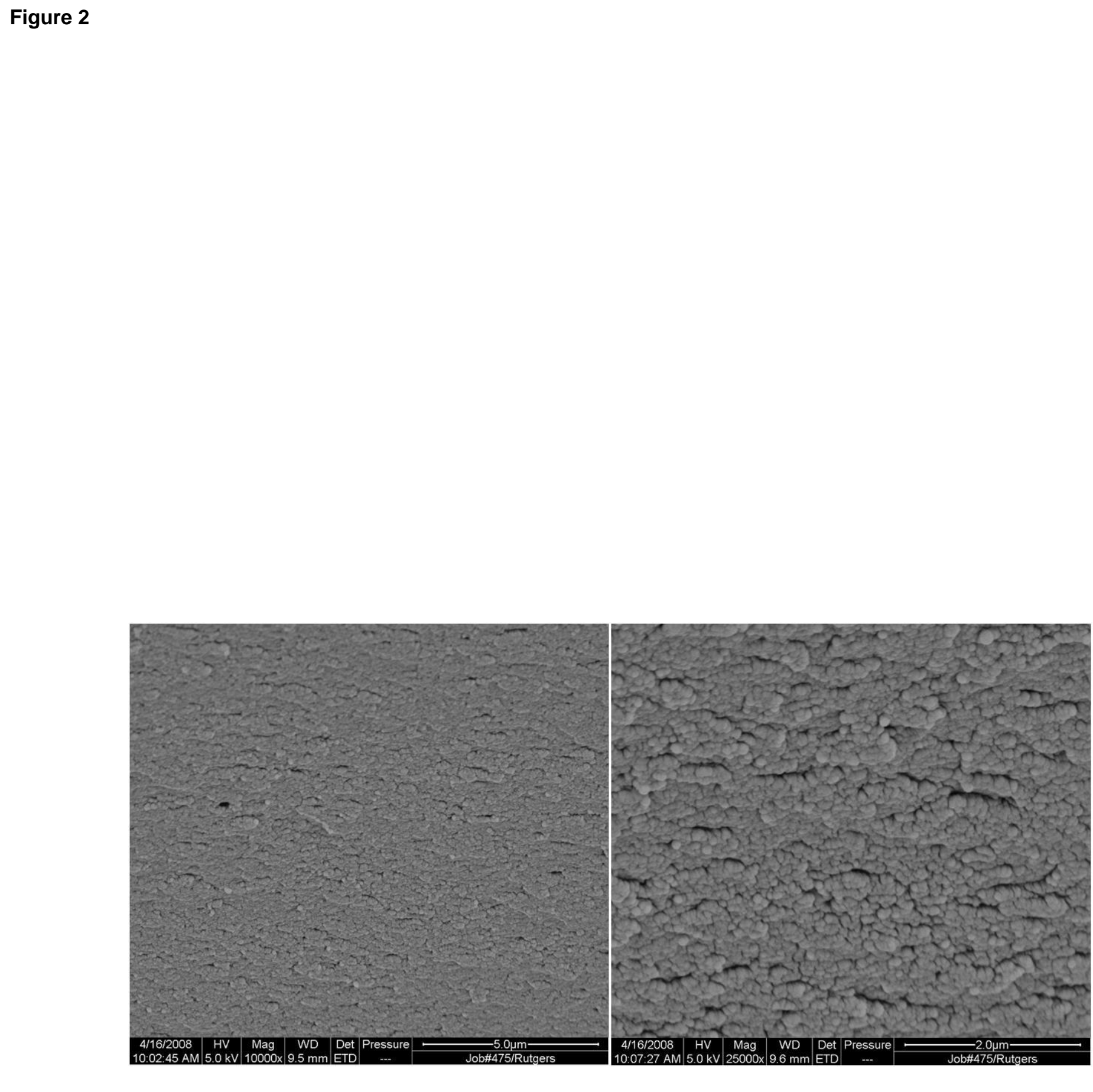
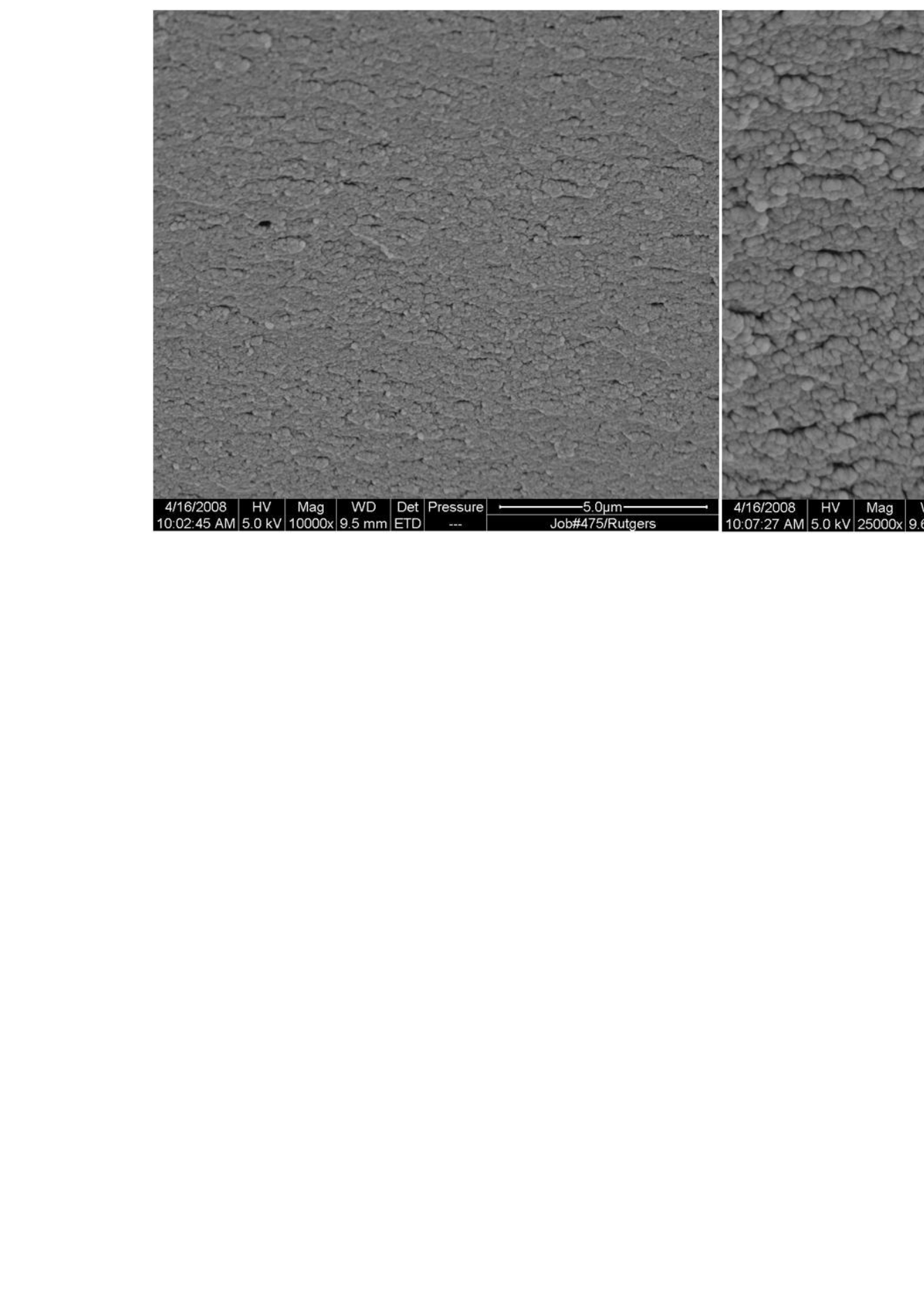

2
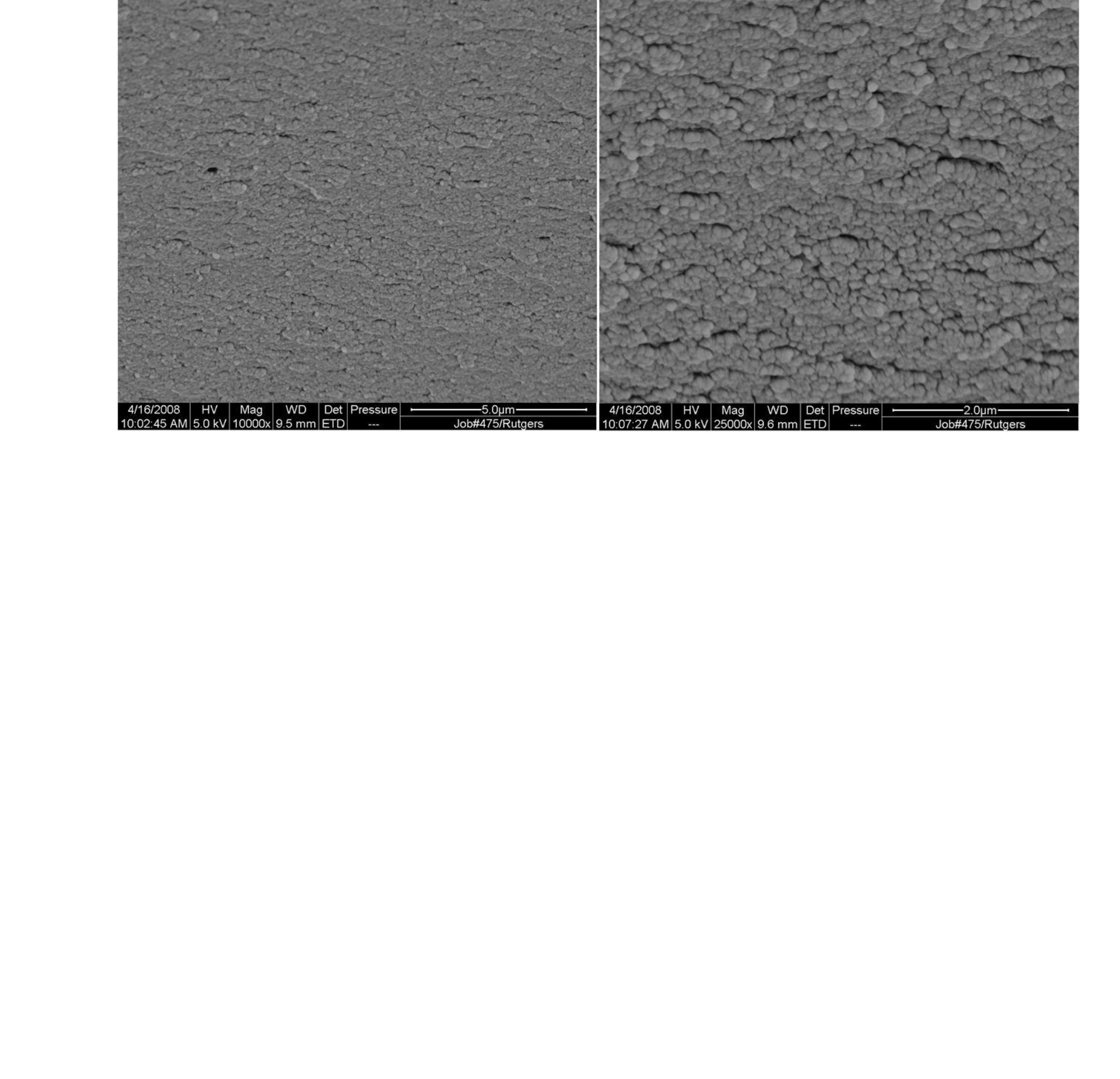

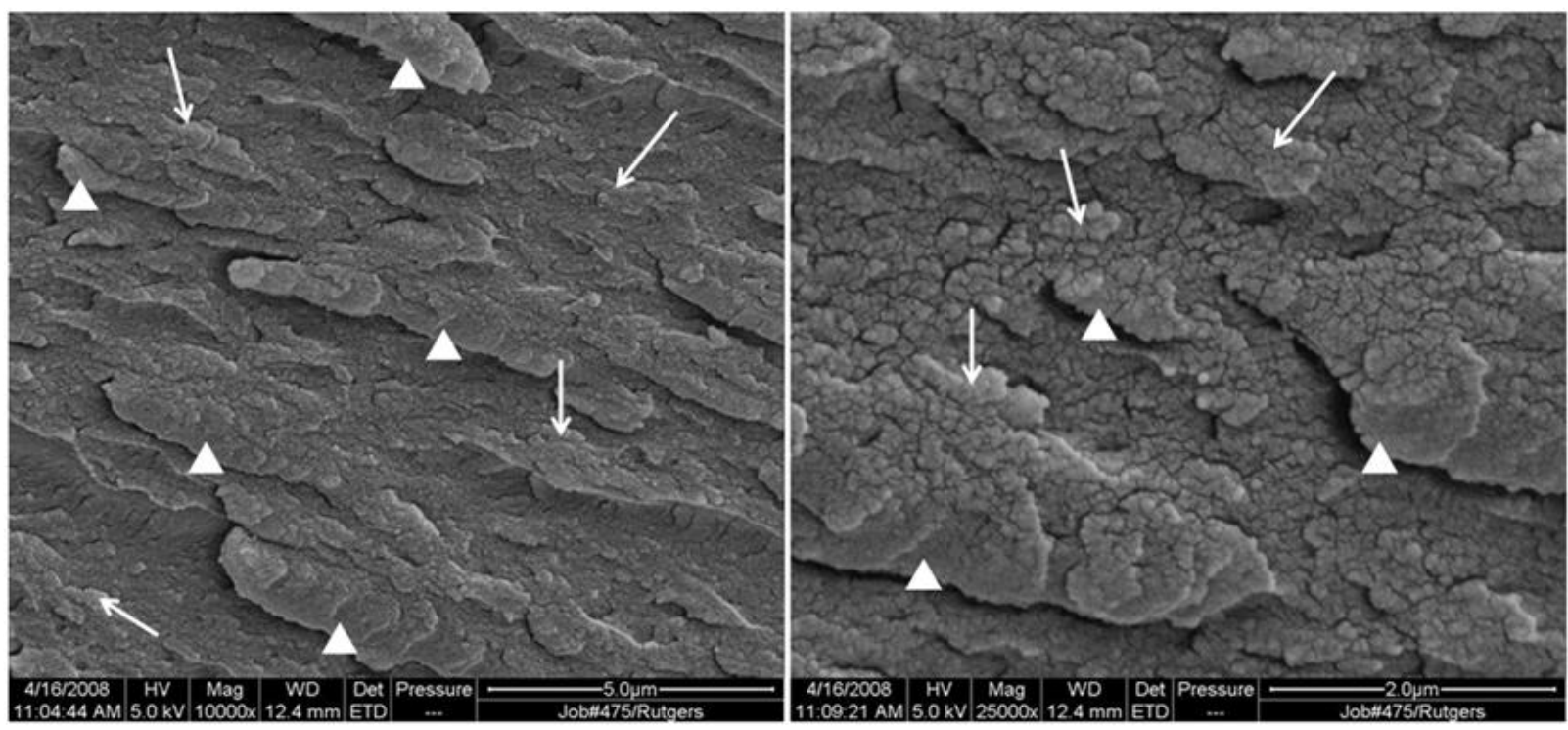

Figure 3

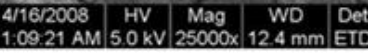

4475/Rutgers 

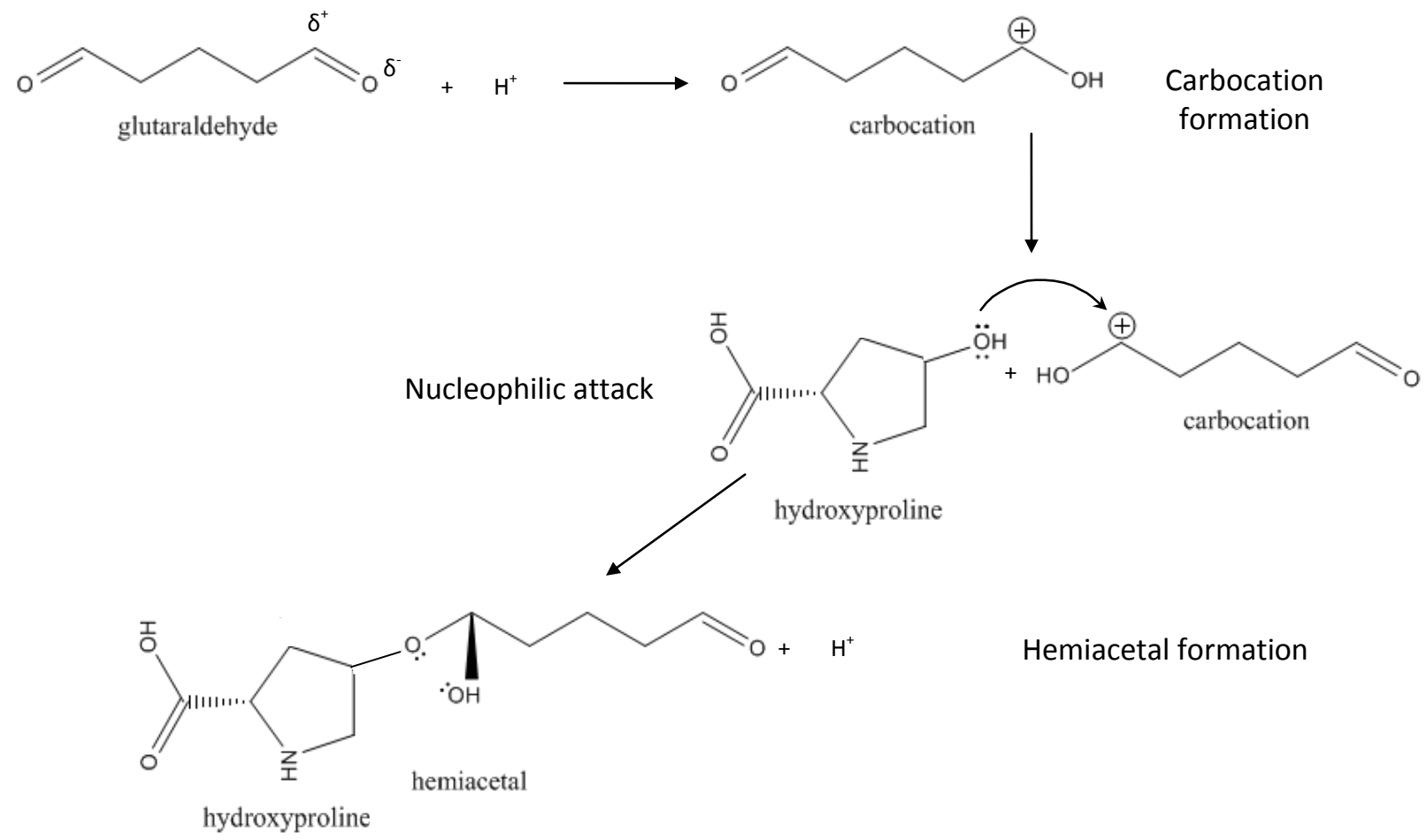


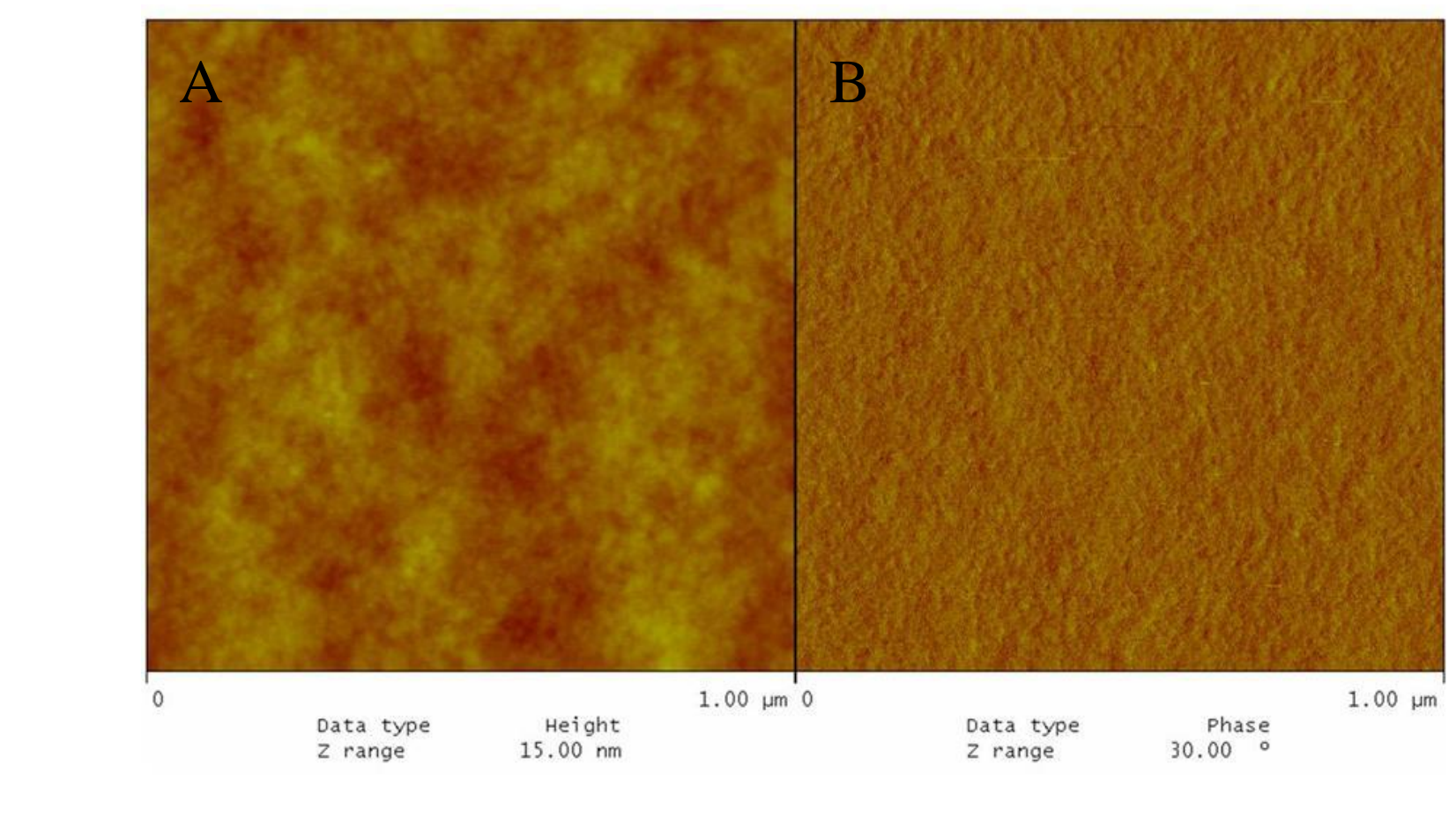

Figure 6

$z$ range

$15.00 \mathrm{~nm}$

$z$ range

$30.00 \%$

\section{Figure 6}

(
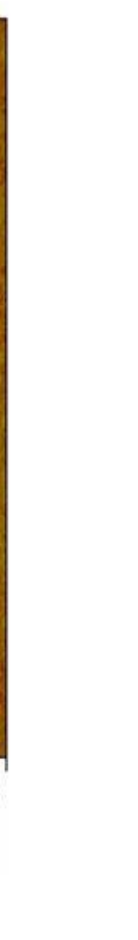

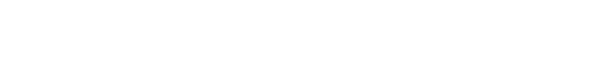




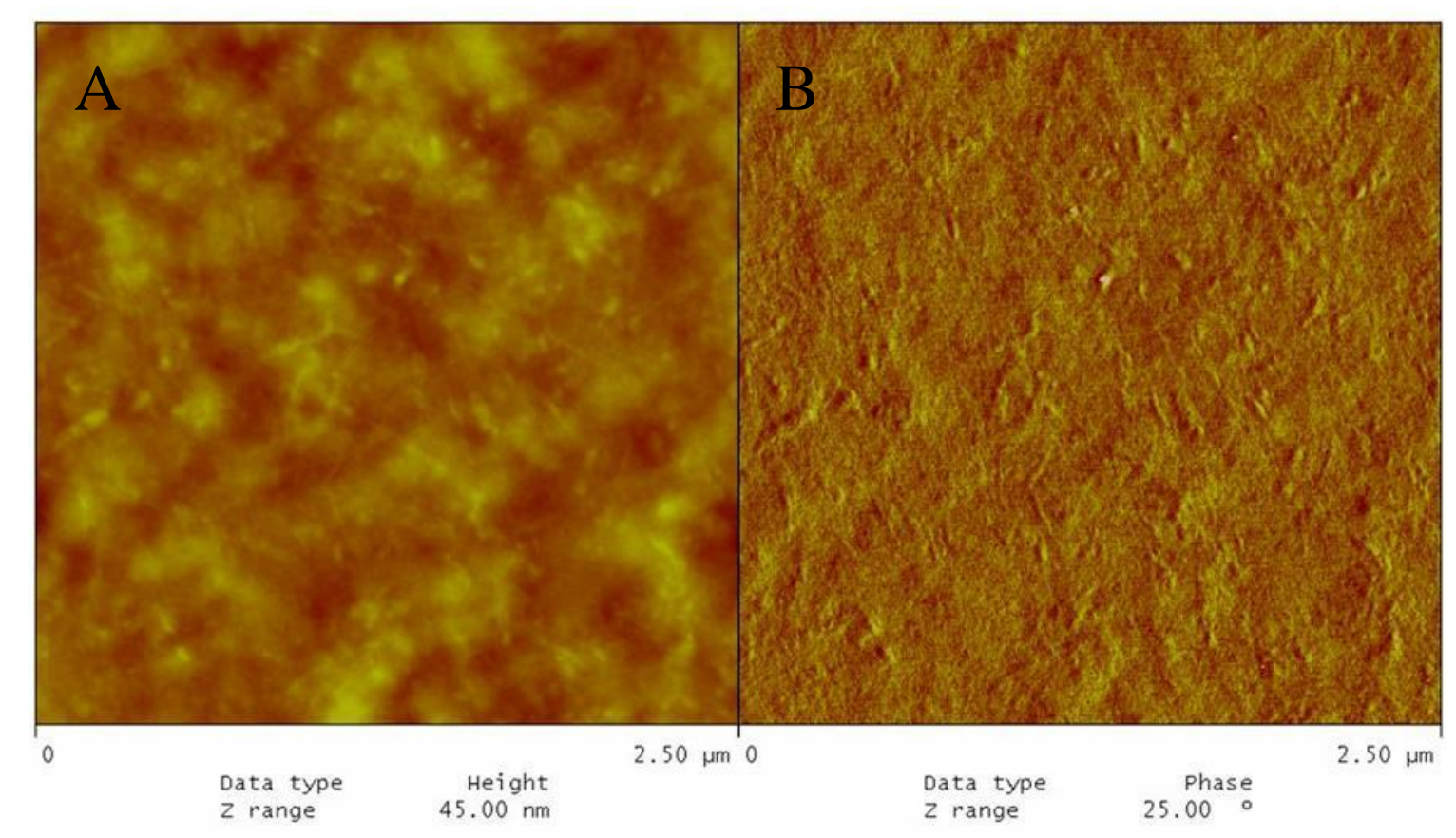

Figure 7

. 


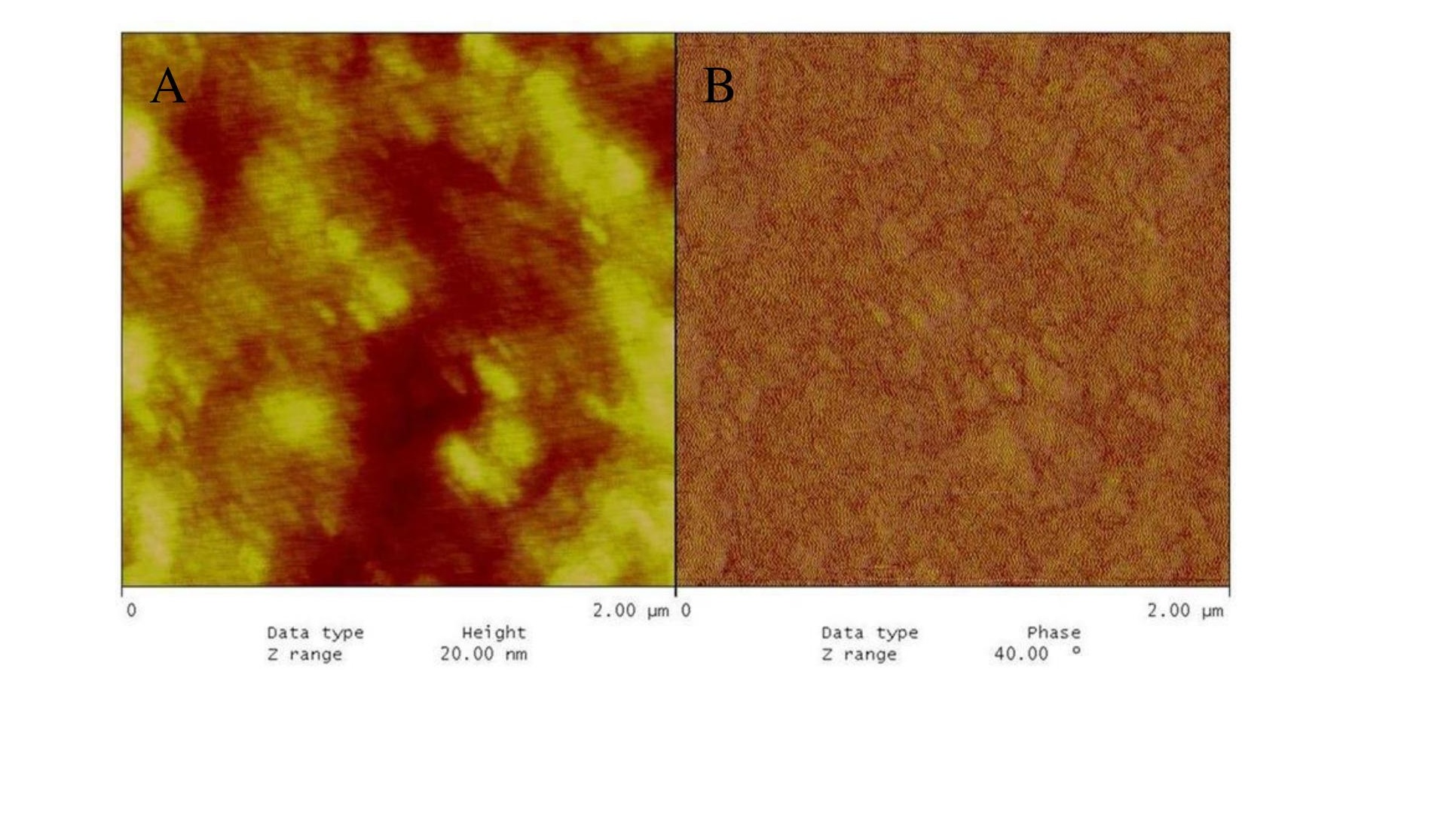

Figure 8

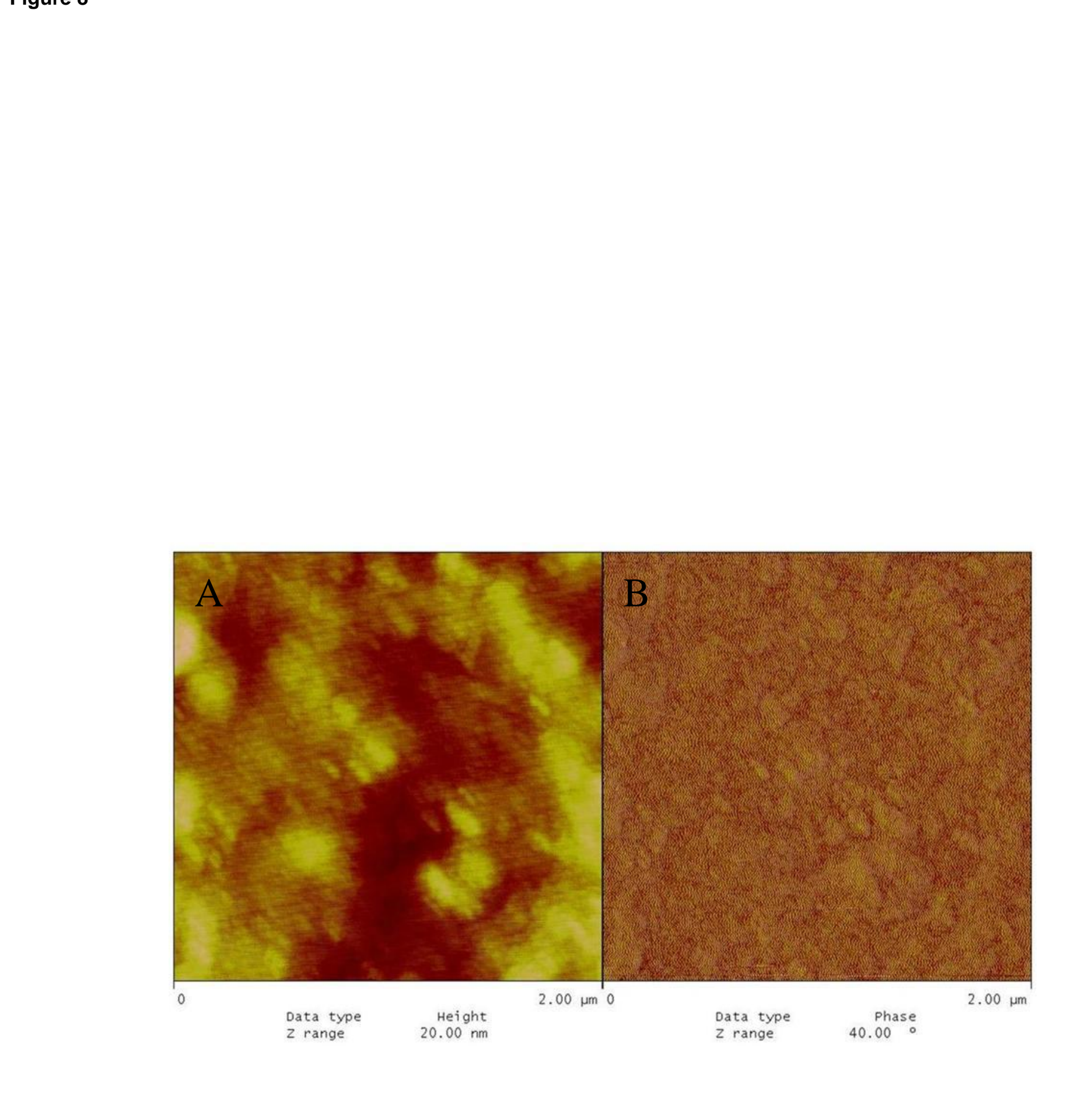

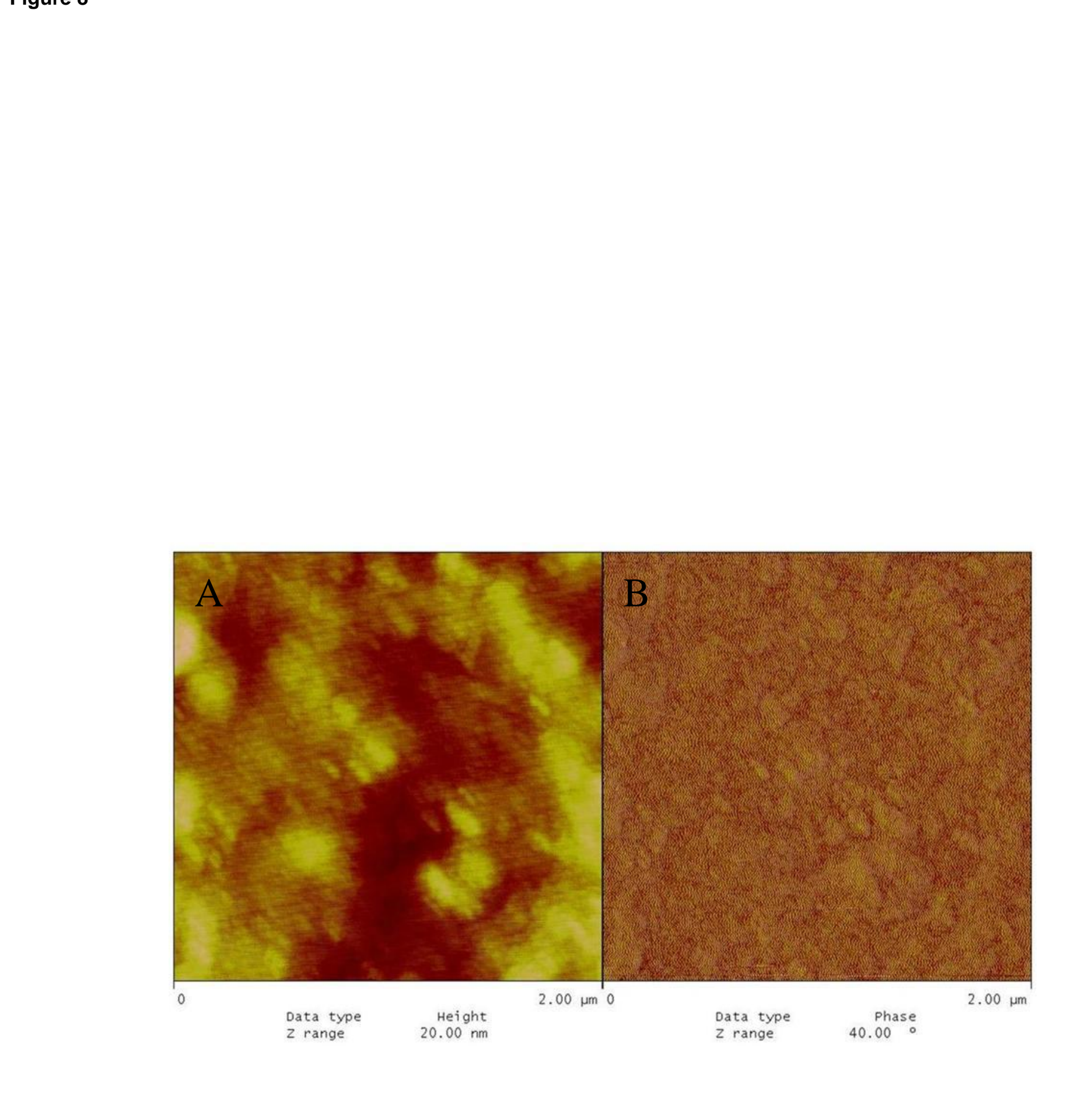

\section{Figure 8}

8

.

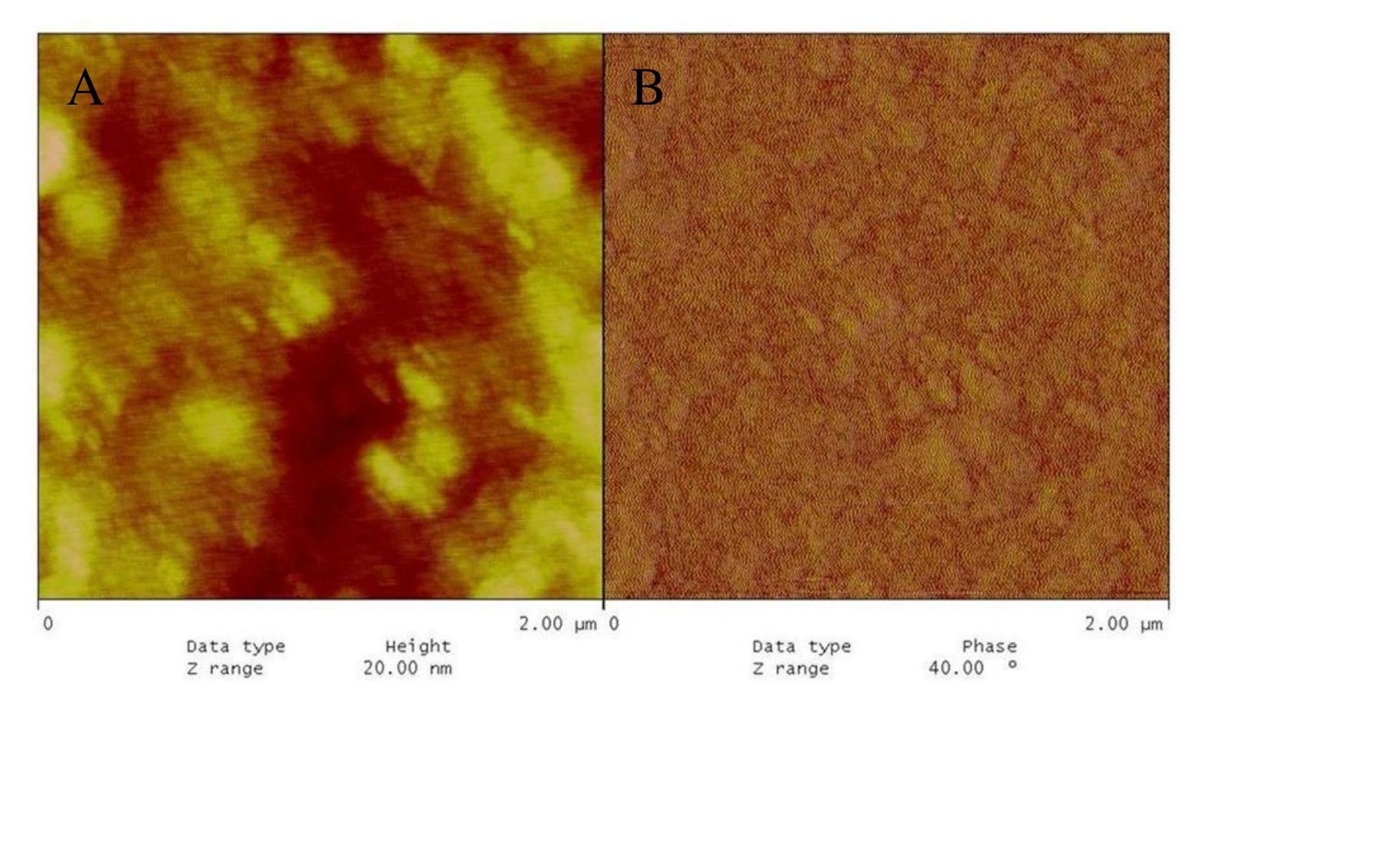

$+$

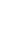

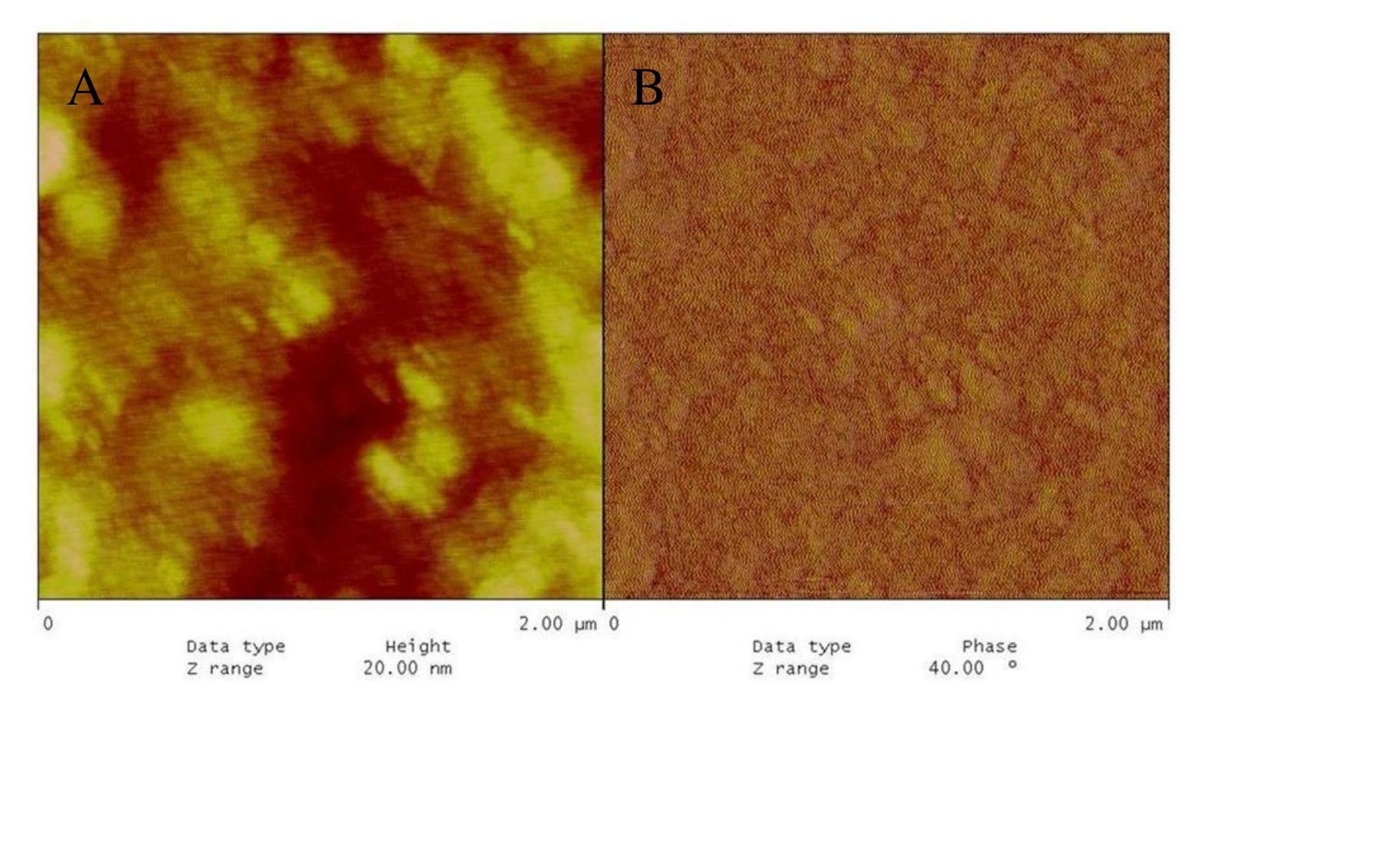

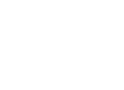
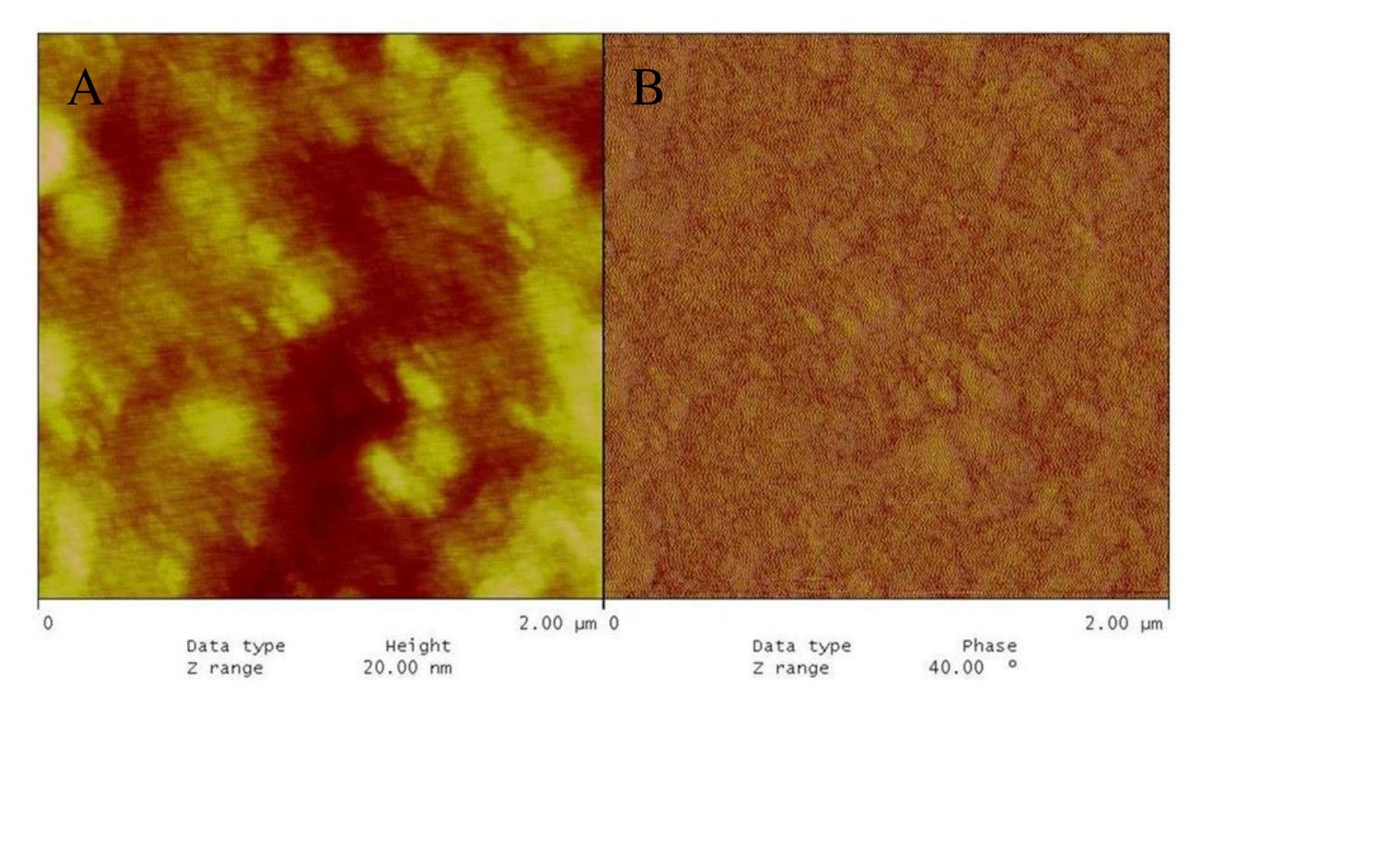


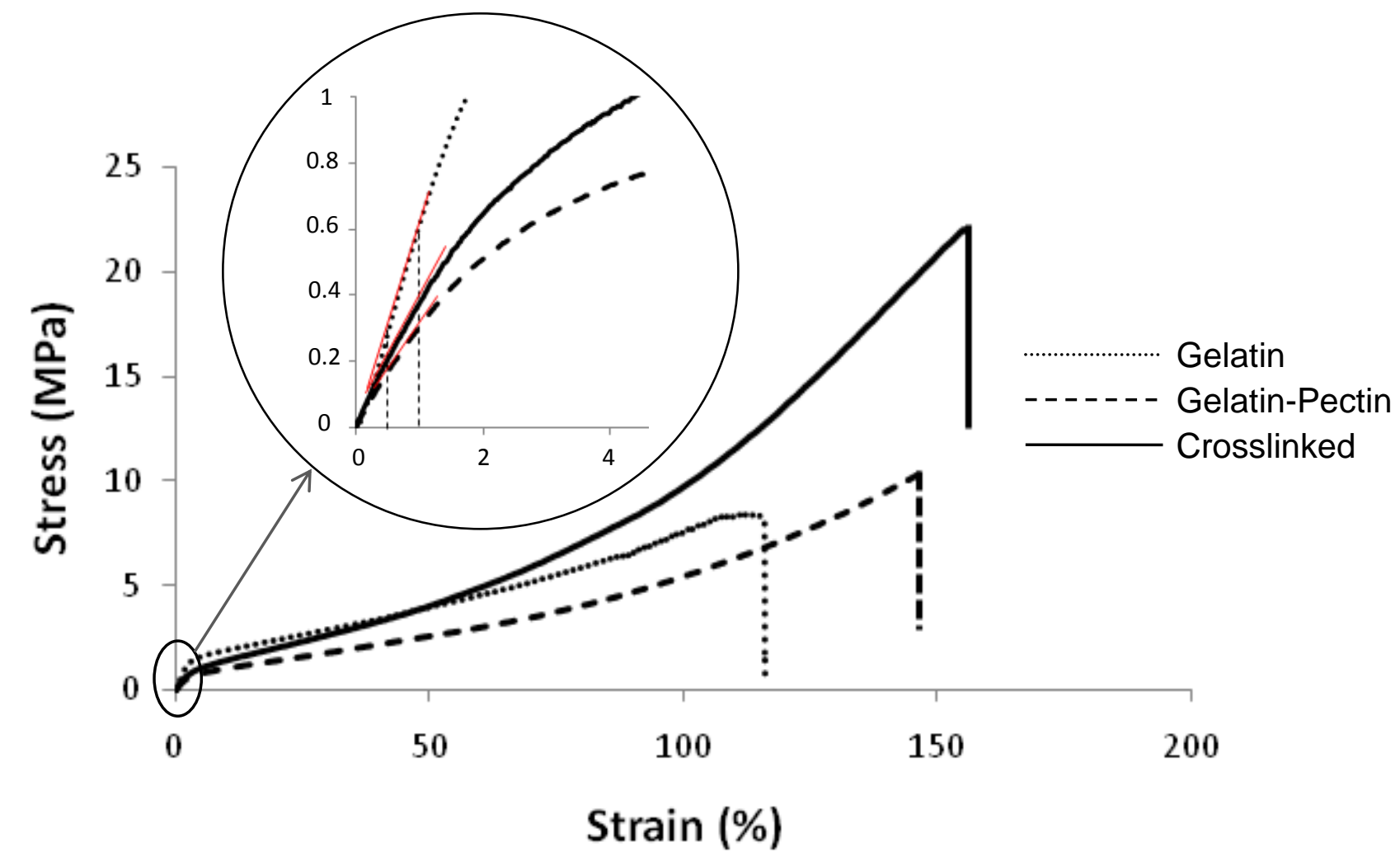

Figure 9 


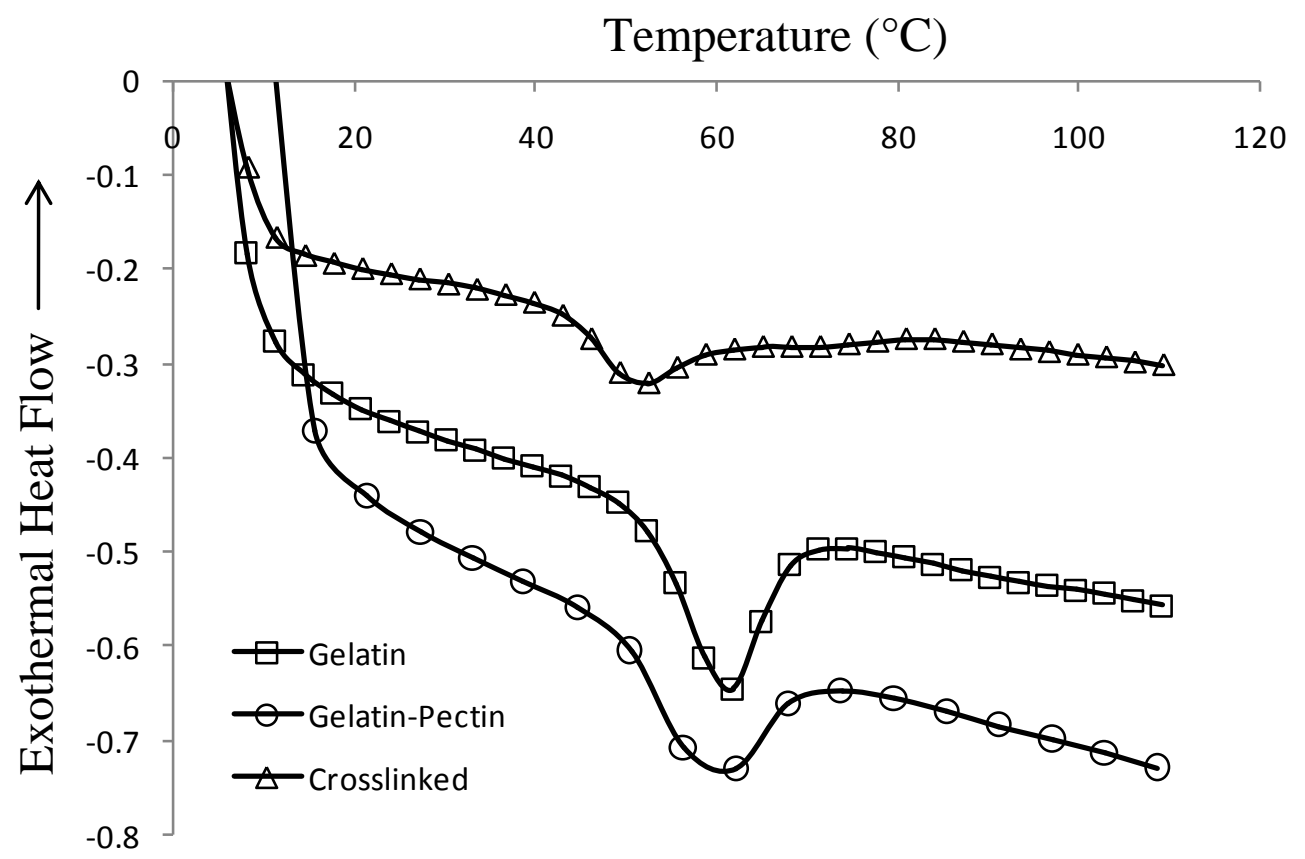


Figure 11

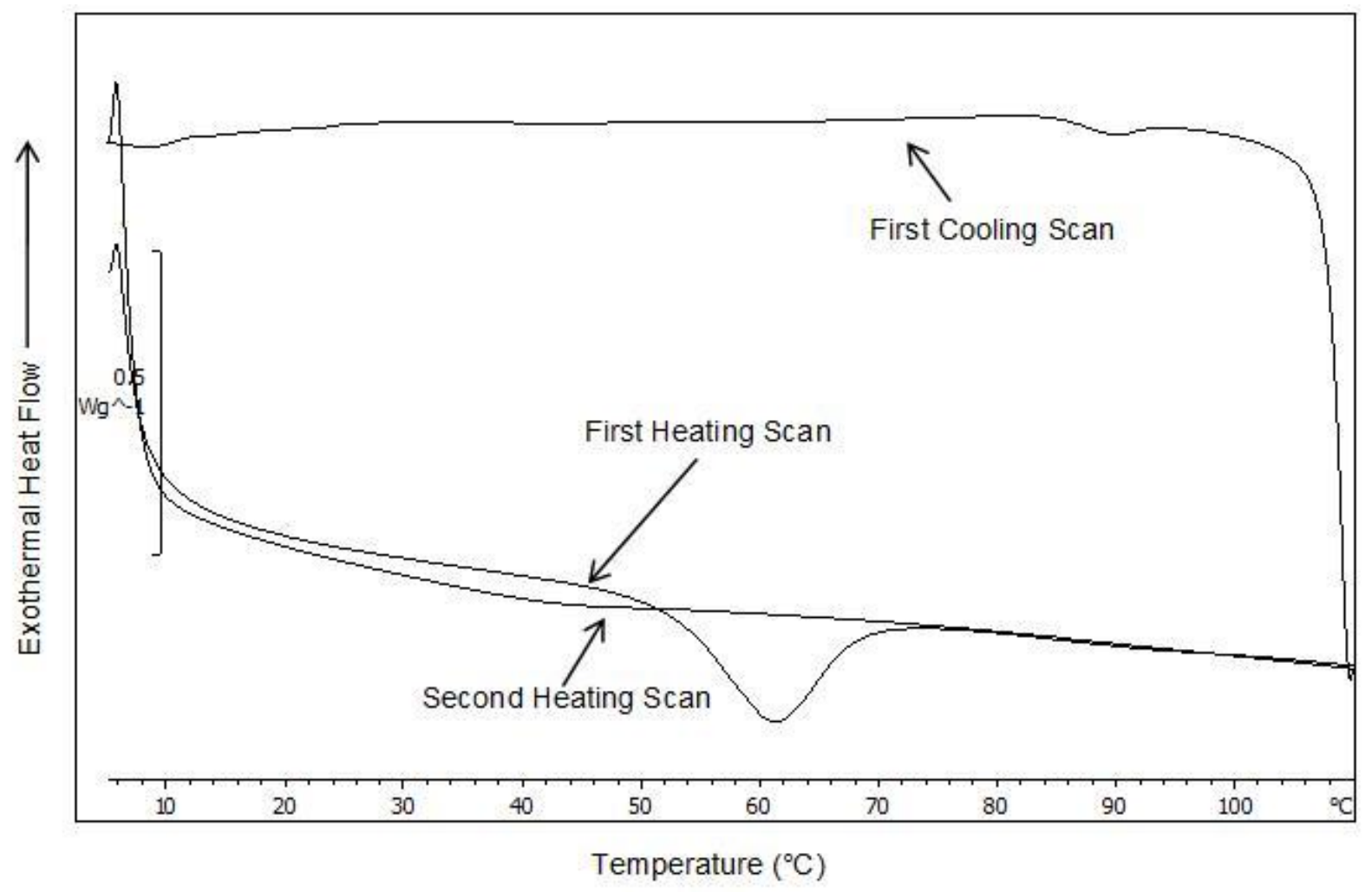


a

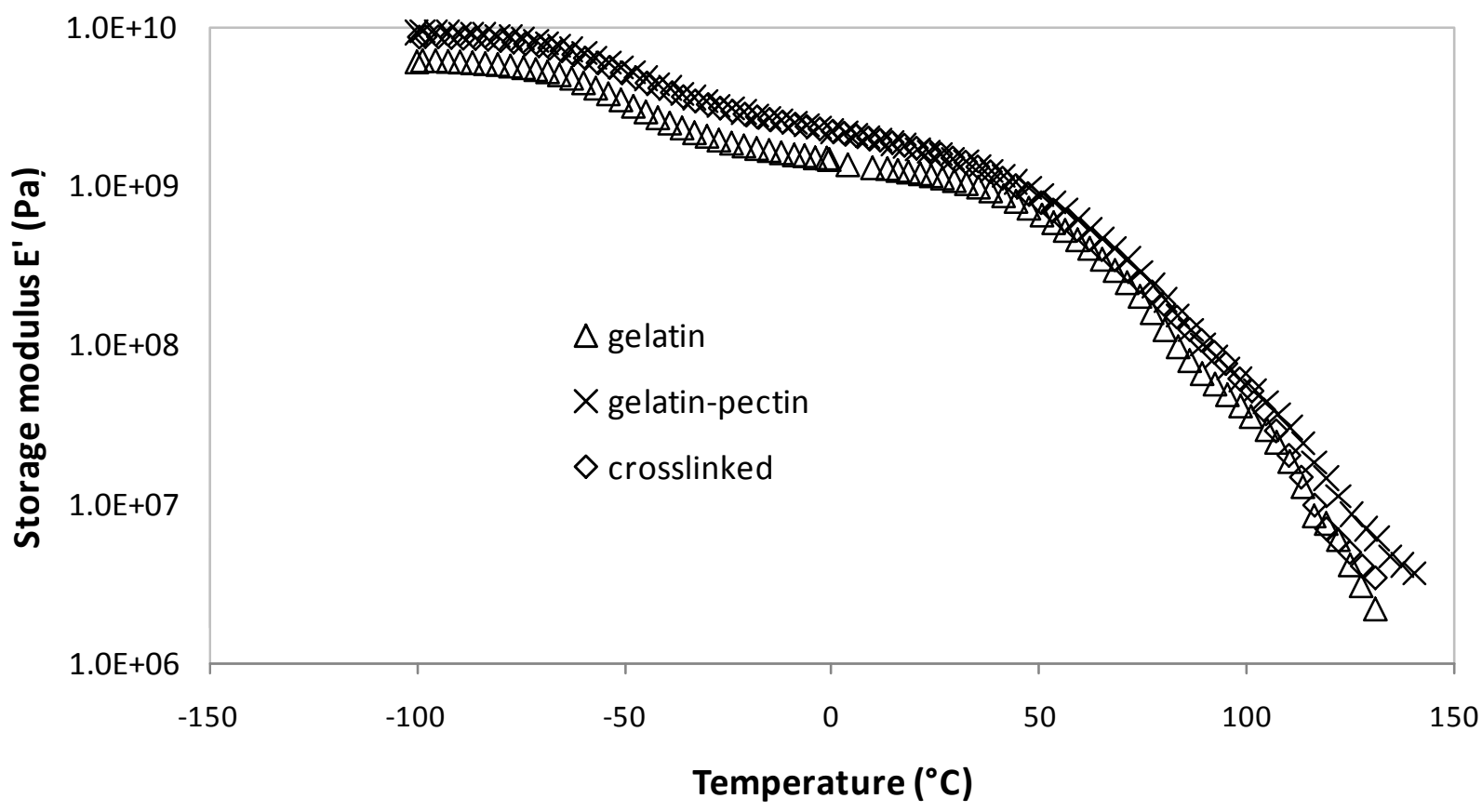

b

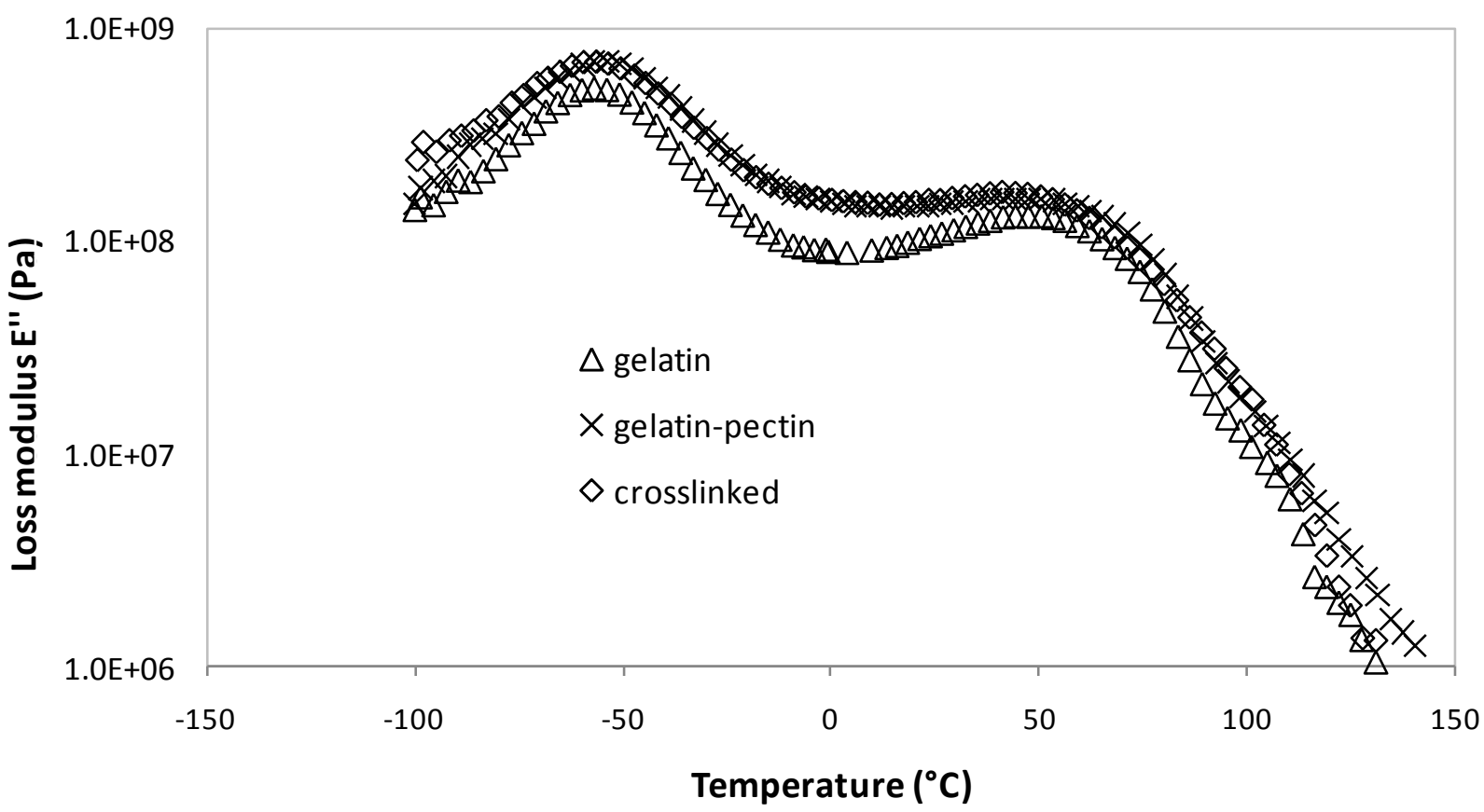




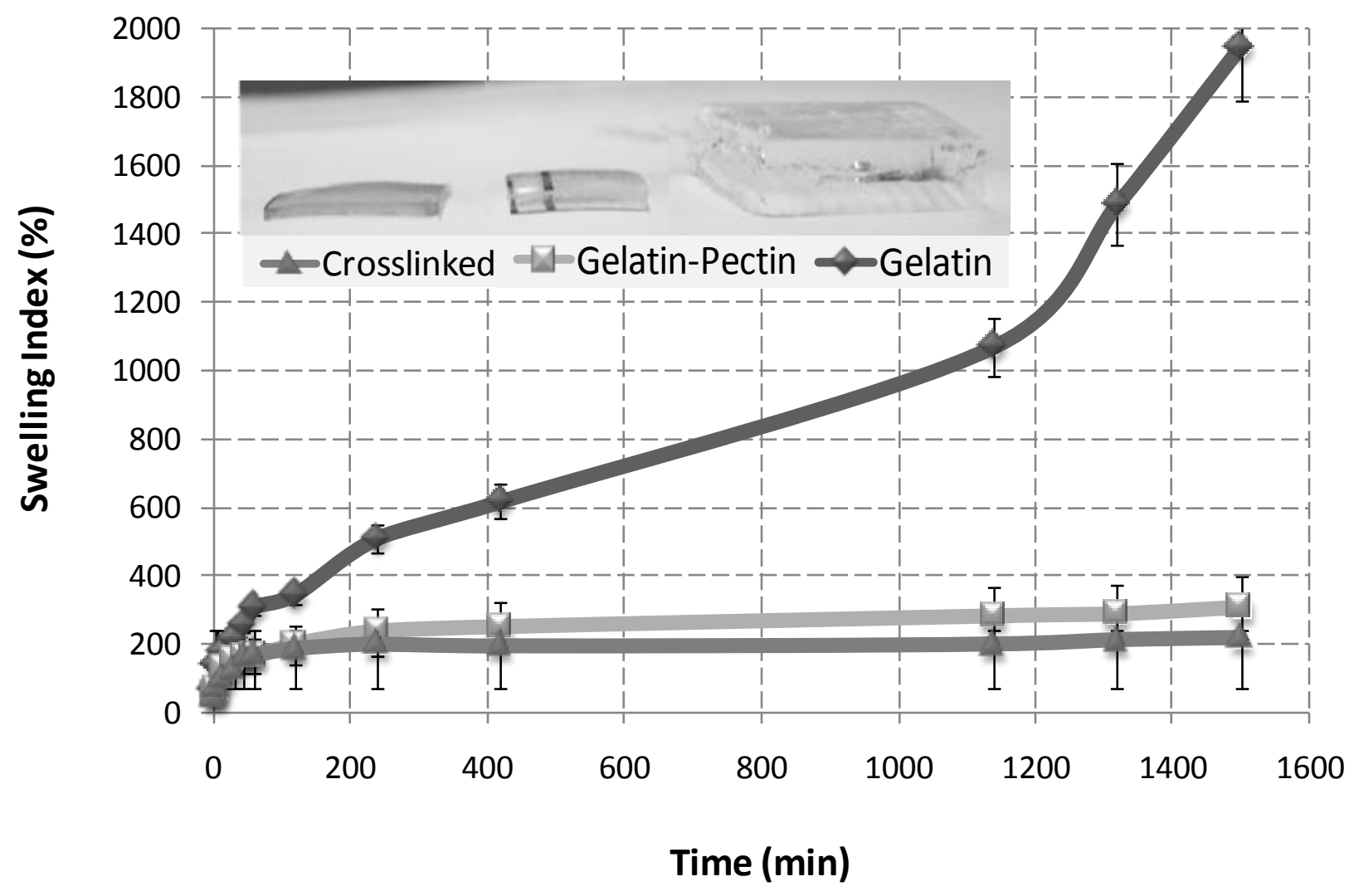


Table 1. Mechanical properties of the three different types of composite films

\section{Parameter}

Formulation

Gelatin

Gelatin-Pectin

Crosslinked

\begin{tabular}{ccc}
\hline $\begin{array}{c}\text { Elastic Modulus } \\
(\mathrm{MPa})\end{array}$ & $\begin{array}{c}\text { Tensile strength } \\
(\mathrm{MPa})\end{array}$ & $\begin{array}{c}\text { Elongation } \\
(\%)\end{array}$ \\
\hline $0.5 \pm 0.12^{\mathrm{a}}$ & $8.22 \pm 0.92^{\mathrm{d}}$ & $115.33 \pm 7.79^{\mathrm{g}}$ \\
$0.28 \pm 0.07^{\mathrm{b}}$ & $11.09 \pm 1.51^{\mathrm{e}}$ & $151.57 \pm 10.24^{\mathrm{h}}$ \\
$0.35 \pm 0.04^{\mathrm{c}}$ & $21.59 \pm 3.74^{\mathrm{f}}$ & $159.1 \pm 16.26^{\mathrm{h}}$ \\
\hline
\end{tabular}

Results are expressed as a mean \pm standard deviation.

Different letters denote statistically significant differences $(\mathrm{p}<0.05)$ between formulations. 